LA W RENCE LIVERMORE NATIONAL LABORATORY

Analysis of the Formation Enthalpy Dataset

C. Kamath

September 19, 2012 
This document was prepared as an account of work sponsored by an agency of the United States government. Neither the United States government nor Lawrence Livermore National Security, LLC, nor any of their employees makes any warranty, expressed or implied, or assumes any legal liability or responsibility for the accuracy, completeness, or usefulness of any information, apparatus, product, or process disclosed, or represents that its use would not infringe privately owned rights. Reference herein to any specific commercial product, process, or service by trade name, trademark, manufacturer, or otherwise does not necessarily constitute or imply its endorsement, recommendation, or favoring by the United States government or Lawrence Livermore National Security, LLC. The views and opinions of authors expressed herein do not necessarily state or reflect those of the United States government or Lawrence Livermore National Security, LLC, and shall not be used for advertising or product endorsement purposes.

This work performed under the auspices of the U.S. Department of Energy by Lawrence Livermore National Laboratory under Contract DE-AC52-07NA27344. 


\title{
Analysis of the Formation Enthalpy Dataset
}

\author{
Chandrika Kamath \\ kamath2@llnl.gov \\ Lawrence Livermore National Laboratory \\ September 14, 2012
}




\section{Contents}

1 Introduction $\quad 1$

2 Description of the data $\quad 2$

2.1 Creating the datasets to describe the compounds . . . . . . . . . . . . . 2

3 Analysis of the periodic table data 3

3.1 Analysis of the $\mathrm{Co} 2 \mathrm{ZnO} 4$ periodic table dataset . . . . . . . . . . . . 4

3.2 Analysis of the $\mathrm{Rh} 2 \mathrm{ZnO} 4$ periodic table dataset $\ldots \ldots \ldots \ldots$

3.3 Analysis of the $\mathrm{Mn} 2 \mathrm{CrO} 4$ periodic table dataset . . . . . . . . . . . . . 7

3.4 Summary of the analysis of periodic table datasets . . . . . . . . . . . 8

4 Analysis of the properties data $\quad 8$

4.1 Analysis of the $\mathrm{Co} 2 \mathrm{ZnO} 4$ properties dataset . . . . . . . . . . . . . . . . 11

4.2 Analysis of the $\mathrm{Rh} 2 \mathrm{ZnO} 4$ properties dataset . . . . . . . . . . . . . . . 12

4.3 Analysis of the $\mathrm{Mn} 2 \mathrm{CrO} 4$ properties dataset . . . . . . . . . . . . . . . 13

4.4 Summary of analysis of properties dataset . . . . . . . . . . . . . . . 14

5 Conclusions and future work $\quad 15$

6 Acknowledgment $\quad 15$

$\begin{array}{lll}\text { A Results for the analysis of properties data for } \mathrm{Co2ZnO} 4 & 17\end{array}$

B Results for the analysis of properties data for $\mathrm{Rh2ZnO4} 23$

C Results for the analysis of properties data for $\mathrm{Mn2CrO4} 29$ 


\section{Introduction}

This report summarizes the work done as part of the MINDES: Data Mining for Inverse Design project [3] to mine the datasets generated by the Center for Inverse Design [1], an Energy Frontier Research Center (EFRC) of the Office of Science, US Department of Energy. In the course of the MINDES project, two datasets were analyzed, one on the formation enthalpy of spinels, and the other on the band gap type of the class of ternary compounds generated at NREL; the former is the focus of this report.

The dataset considered in this analysis is from computer simulations of spinel materials. Given a spinel material represented as $\mathrm{A} 2 \mathrm{BO} 4$, where $\mathrm{A}$ and $\mathrm{B}$ are atomic species (O represents oxygen), with the ratio of atoms $\mathrm{A}: \mathrm{B}: \mathrm{O}=16: 8: 32$, simulations are run to obtain the formation enthalpy of a new compound derived from A2BO4 by introducing an impurity in the form of either a vacancy or a substitution in one of the locations of the A, B, or O atoms. The focus of the analysis is to determine, if, given a sample of compounds derived from a spinel material and the corresponding formation enthalpy for these compounds, is it possible to predict the formation enthalpy of a compound derived from the same spinel material using an impurity not considered previously? We expect that the ideas explored in the course of this study will also apply in the analysis of other physical properties of compounds.

This analysis falls in the broad area of design of computer experiments [2], where an ensemble of simulations is used to guide physical experiments and gain insights into the design space which maps the inputs of the simulations to the output(s). As the simulations are often computationally expensive, the ensemble must be carefully designed to gain the greatest insights into the physical phenomenon of interest using as few simulations as possible. A possible solution is to consider an incremental approach where we analyze the input/output data from the simulations that have already been run to identify the next set of simulations such that these new simulations would add the greatest insights, by either refining the original data set in a region of interest, or exploring new regions in the design space.

Therefore, in the context of our problem, we can analyze the simulations that have been run thus far to understand what properties of the elements comprising a compound are relevant to, and predictive of, the formation enthalpy. By combining this information with physics insights, we can suitably create other compounds and expect that their simulation will likely indicate the compounds to have the formation enthalpy predicted by the analysis.

In this report, we start with a description of the data available for analysis in Section 2, and briefly discuss the two types of datasets - the periodic table dataset and the properties dataset - that we can create from the data provided. For the three spinel materials considered in this work - $\mathrm{Co} 2 \mathrm{ZnO} 4, \mathrm{Rh} 2 \mathrm{ZnO} 4$, and $\mathrm{Mn} 2 \mathrm{CrO} 4$ - we discuss the analysis results for the periodic table dataset in Section 3 and the properties dataset in Section 4. We conclude with a brief summary and some thoughts for future work.

Disclaimer - This report is written from the point of view of a data miner, not a materials scientist, a physicist, or a chemist. The analysis is purely data driven and is not influenced by any domain-specific biases. Also, any conclusions drawn must be interpreted with care as the analysis reflects the characteristics and quality of the data provided; the availability of additional data may change the results. 


\begin{tabular}{|c|c|c|c|c|}
\hline name & symbol & group & column & row \\
\hline mulliken jaffe & single bond radius & pauling & molar volume & atomization \\
\hline sound velocity & sanderson & atomic weight & critical temperature & triple bond radius \\
\hline melting point & allen & thermal conductivity & electrical resistivity & vaporization \\
\hline atomic number & rigidity modulus & covalent radius & fusion & pettifor \\
\hline bulk modulus & poisson ratio & van der waals radius & electron affinity & boiling point \\
\hline density & double bond radius & allred rochow & young modulus & thermal expansion \\
\hline atomic radius & orbital radii s & orbital radii o & first ionization energy & \\
\hline
\end{tabular}

Table 1: Information available for the different atomic species, including the material properties. The group, row, and column refer to the location of the species in the periodic table. Not all properties are available for all species.

\section{Description of the data}

There are three types of data available for the analysis described in this report:

- Formation enthalpy values: These are available for compounds generated from three spinel materials: $\mathrm{Co} 2 \mathrm{ZnO} 4, \mathrm{Cr} 2 \mathrm{ZnO} 4, \mathrm{Mn} 2 \mathrm{CrO} 4$. These compounds are created by introducing an impurity, which can be either a vacancy at one of the locations in the spinel structure or a substitution of one atom of an atomic species by another atomic species. These impurities can be at different charge states, with a different formation enthalpy associated with each charge state. For Co2ZnO4, the dataset has 53 compounds, while $\mathrm{Cr} 2 \mathrm{ZnO} 4$ has 52 compounds and $\mathrm{Mn} 2 \mathrm{CrO} 4$ has 49 compounds.

- Structure data; These provide the locations of the different atoms in 3-D space. Some of the structure files are missing as $\mathrm{Co} 2 \mathrm{ZnO} 4$ has 32 structure files and $\mathrm{Cr} 2 \mathrm{ZnO} 4$ has 47 structure files, though the two materials have 53 and 52 compounds, respectively. There were no files provided for the $\mathrm{Mn} 2 \mathrm{CrO} 4$ compounds. The structures files are not used in the present analysis so the missing files are not an issue. However, as observed later in the report, the structure data may be relevant in future work.

- Material properties: These are the properties of the different atomic species as listed in Table 1. However, not all properties are available for all atomic species.

\subsection{Creating the datasets to describe the compounds}

Our goals in this analysis are to identify the atomic properties that are predictive of the formation enthalpy and to determine if we can predict the enthalpy for a new compound. We are also interested in seeing how much of the knowledge gained in the analysis of one spinel material carries over to another.

To accomplish these goals, we need to convert the data available into an appropriate form so that each compound is represented by a series of properties (called "features" in the data mining literature) and its formation enthalpy. Thus, for a spinel material, say Co2ZnO4, each 
compound obtained by adding an impurity would represent a row of a table, with the columns representing the properties describing the compound and its formation enthalpy.

Note: In our work, we consider the compounds associated with the three spinel materials as separate datasets. We will refer to the original compound as the "spinel material" or just "material" (e.g., Mn2CrO4) and compounds derived from this as "derived compounds" or just "compounds" (e.g., Co2ZnO4_Mg_on_Co_0 which represents the spinel material Co2ZnO4, with one Co atom replaced by $\mathrm{Mg}$, in charge state 0 ).

There are two challenges in creating the table for each material. First, how do we define the columns of the table so we can represent both vacancies and substitutions in similar manner, that is, each column in the table represents the same feature for both types of impurities? Second, if we use the material properties (Table 1) to represent each atomic species, how do we suitably modify the properties for a species if one of its atoms is missing or replaced by another? For example, in $\mathrm{Co} 2 \mathrm{ZnO} 4$, we have $8 \mathrm{Zn}$ atoms, $16 \mathrm{Co}$ atoms, and $32 \mathrm{O}$ atoms, so a reasonable first attempt is to have three groups of columns, one representing the properties for each atomic species. If a Co atom is replaced by a Ca atom, then we have introduced a fourth species. But, if we replace a Co atom by a Zn atom, we have introduced an impurity in a specific location (a Co atom), without changing the number of atomic species in the compound. Similarly, a vacancy in any one of the three species would reduce the number of atoms for that species, while the number of species remained three. To accommodate all these cases in a consistent manner and to manage the large number of properties representing each atomic species, we created two types of datasets for each spinel material:

- Periodic table dataset: In this smaller dataset, we consider just the periodic table information for each species to determine if we can learn any patterns from the data. More details and the results of the analysis are described in Section 3.

- Properties dataset: Here, instead of the location of the impurity in the periodic table, we consider the properties associated with the constituent atomic species. The intent is to determine if the values of the properties are reflective of the formation enthalpy. More details and the results of the analysis are described in Section 4.

\section{Analysis of the periodic table data}

In this dataset, we consider just the periodic table information for each species; this includes five quantities: its symbol, the number of atoms, and the group, row, and column of the periodic table for the species.

To accomodate compounds created by either a vacancy or a substitution in a single table, we consider the table to be formed by a group of columns describing each of the three main species in the spinel ( $\mathrm{Co}, \mathrm{Zn}$, and $\mathrm{O}$ in the case of $\mathrm{Co} 2 \mathrm{ZnO} 4)$, and a group of columns describing the impurity associated with each species. Since there is only one impurity being considered in our analysis (an atom is either removed or substituted by another), there is some redundancy in this representation. For example, in the $\mathrm{Co} 2 \mathrm{ZnO} 4$ data, if a Co atom is replaced by another species, we know that there will not be any impurity associated with the $\mathrm{Zn}$ or $\mathrm{O}$ species. In such cases, the "impurity" is identified by the sympol "XYZ" and all its periodic table information is set to zero. Further, in our dataset, the only "impurity" associated with the O 


\begin{tabular}{|c|c|c|c|}
\hline Compound ID & natoms Zn & symbol imp_Zn & natoms imp_Zn \\
\hline PTgroup imp_Zn & PTrow imp_Zn & PTcol imp_Zn & natoms Co \\
\hline symbol imp_Co & natoms imp_Co & PTgroup imp_Co & PTrow imp_Co \\
\hline PTcol im_Co & natoms O & charge state & fenthalpy \\
\hline
\end{tabular}

Table 2: The information in the periodic table (PT) dataset using Co2ZnO4 as the spinel material. The periodic table information includes the group, the row, and the column for the atomic species. "Imp_XX" represents the information for the species which acts as the impurity for XX. If there is no such species, it is represented by the symbol "XYZ" and all the periodic table information is set to zero. The compound ID is a string generated by concatenating the symbols for the spinel material with information on the impurity and the charge state, e.g., Co2ZnO4_Mg_on_Co_O.

atom is a vacancy. This is handled simply by reducing the value of the feature representing the number of $\mathrm{O}$ atoms; there are no columns to indicate an impurity associated with the $\mathrm{O}$ species.

We also observe that the periodic table information for the three main species (Co, Zn, and $\mathrm{O}$ in the case of $\mathrm{Co} 2 \mathrm{ZnO} 4$ ), are the same for all compounds, so we do not need to include the columns for these features in the table. With these simplifications, the periodic table dataset contains the variables listed in Table 2 .

Caveat: These observations in the next three sections are based on a relatively small dataset (53 examples for Co2ZnO4, 52 for $\mathrm{Rh} 2 \mathrm{ZnO} 4$, and 49 for $\mathrm{Mn} 2 \mathrm{CrO} 4$ ). They are more qualitative in nature and may reflect the set of samples in the data rather than the physics. Therefore, any interpretation and extrapolation must be done with care.

\subsection{Analysis of the $\mathrm{Co2ZnO4}$ periodic table dataset}

For $\mathrm{Co} 2 \mathrm{ZnO} 4$, we first simplified the analysis by assigning a label of "low", "med", or "high" to each compound based on the value of formation enthalpy, which varies from -0.08 to 6.6 for the 53 compounds. The first attempt selected thresholds that split the 53 examples into three equal groups. However, this led to some cases which were close to the threshold, which was then changed so there were no examples near the threshold values. This led to compounds with formation enthaply less than or equal to 1.4 to be labeled "low" (22 examples) and those higher than 3.3 to be labeled as "high" (14 examples) with the remaining 17 examples being labeled "med".

We make the following observations on the data:

1. It appears that the substitutions (for both Co and $\mathrm{Zn}$ ) are from either column 2 or row 4 of the periodic table.

2. When the compounds are listed in increasing value of f-enthalpy, examples with higher f-enthalpy tend to have negative charge state, while those with lower enthalpy tend to have positive charge states.

3. When the compounds are listed in increasing value of f-enthalpy, examples with lower f-enthalpy (with labels "low" or "med") have either a substitution in Co or a substitution 


\begin{tabular}{|c|c|c|c|c|c|c|}
\hline Compound ID & impurity & PT row & PT col & charge state & f-enthalpy & label \\
\hline Co2ZnO4_Mg_on_Co_0 & $\mathrm{Mg}$ & 3 & 2 & 0 & 0.700634 & low \\
\hline Co2ZnO4_Mg_on_Co_-1 & $\mathrm{Mg}$ & 3 & 2 & -1 & 1.005975 & low \\
\hline Co2ZnO4_Be_on_Co_0 & $\mathrm{Be}$ & 2 & 2 & 0 & 1.217644 & low \\
\hline Co2ZnO4_Be_on_Co_-1 & $\mathrm{Be}$ & 2 & 2 & -1 & 1.321137 & low \\
\hline Co2ZnO4_Ca_on_Co_0 & $\mathrm{Ca}$ & 4 & 2 & 0 & 1.883827 & med \\
\hline Co2ZnO4_Ca_on_Co_-1 & $\mathrm{Ca}$ & 4 & 2 & -1 & 2.475118 & med \\
\hline Co2ZnO4_Sr_on_Co_0 & $\mathrm{Sr}$ & 5 & 2 & 0 & 3.266471 & med \\
\hline Co2ZnO4_Sr_on_Co_-1 & $\mathrm{Sr}$ & 5 & 2 & -1 & 3.951836 & high \\
\hline Co2ZnO4_Ba_on_Co_0 & $\mathrm{Ba}$ & 6 & 2 & 0 & 5.334465 & high \\
\hline Co2ZnO4_Ba_On_Co_-1 & $\mathrm{Ba}$ & 6 & 2 & -1 & 6.102002 & high \\
\hline
\end{tabular}

Table 3: Listed in increasing value of f-enthalpy, the compounds created from Co2ZnO4 by substituting Co with an element from column 2 of the periodic table (PT).

in Zn. The first compound resulting from a vacancy in this ordering of f-enthalpy values occurs at index 27 (out of 53). Note that the converse is not true, that is, a substitution in Co or a substitution in $\mathrm{Zn}$ does not result in a lower f-enthalpy.

4. Equivalently, if the impurity is a vacancy in Co, or a vacancy in $\mathrm{Zn}$, or a vacancy in $\mathrm{O}$, then the f-enthalpy is "med" or "high". The converse is not true as "med" or "high" f-enthalpy can result from substitution impurities.

5. There is one compound with negative f-enthalpy, which is a Cr substitution on Co.

6. For a given species which substitutes a Zn atom, the ones with lower charge state have a higher enthalpy (i.e., if we take the compounds where species $\mathrm{X}$ replaces a $\mathrm{Zn}$ atom and arrange them by decreasing charge state, the order would lead to increasing f-enthalpy values). However, this is not always the case when we consider species which substitute a Co atom.

7. When we consider compounds obtained by substituting Co with an element from column 2 of the periodic table, we observe that the f-enthalpy increases as we go down the column of the periodic table (with the exception of Be, which appears out of order) as shown in Table 3. Note also that, for a species, the lower charge state has higher f-enthalpy.

8. Similarly, when we consider compounds obtained by substituting Zn with an element from column 2 of the periodic table, we observe that the f-enthalpy increases as we go down the column of the periodic table (with the exception of Be, which appears out of order) as shown in Table 4. In this case, the only charge state is zero.

\subsection{Analysis of the $\mathrm{Rh} 2 \mathrm{ZnO} 4$ periodic table dataset}

For Rh2ZnO4, we simplified the analysis by assigning a label of "low", "med", or "high" to each compound based on the value of formation enthalpy, which varies from -2.28 to 12.2 for 


\begin{tabular}{|c|c|c|c|c|c|c|}
\hline Compound ID & impurity & PT row & PT col & charge state & f-enthalpy & label \\
\hline Co2ZnO4_Mg_on_Zn_0 & $\mathrm{Mg}$ & 3 & 2 & 0 & 0.109360 & low \\
\hline Co2ZnO4_Be_on_Zn_0 & $\mathrm{Be}$ & 2 & 2 & 0 & 0.416459 & low \\
\hline Co2ZnO4_Ca_on_Zn_0 & $\mathrm{Ca}$ & 4 & 2 & 0 & 1.011856 & low \\
\hline Co2ZnO4_Sr_on_Zn_0 & $\mathrm{Sr}$ & 5 & 2 & 0 & 1.989687 & med \\
\hline Co2ZnO4_Ba_on_Zn_0 & $\mathrm{Ba}$ & 6 & 2 & 0 & 3.521112 & high \\
\hline
\end{tabular}

Table 4: Listed in increasing value of f-enthalpy, the compounds created from Co2ZnO4 by substituting $Z n$ with an element from column 2 of the periodic table (PT).

the 52 compounds (there is a large gap between the highest f-enthalpy and the next highest at 6.5). As with $\mathrm{Co} 2 \mathrm{ZnO} 4$, the first attempt at selecting thresholds split the 52 examples into three equal groups. However, this led to some cases which were close to the threshold. So, the threshold was changed so there were no examples near the threshold values. This led to compounds with formation enthaply less than or equal to 1.15 to be labeled "low" (19 examples) and those higher than 2.9 to be labeled as "high" (12 examples) with the remaining 21 examples being labeled "med".

We make the following observations on this dataset:

1. It appears that the substitutions (for both $\mathrm{Rh}$ and $\mathrm{Zn}$ ) are from column 2 or row 4 of the periodic table. There are two exceptions - Rh on $\mathrm{Zn}$ substitutions at charge state 0 and 1.

2. When the compounds are listed in increasing value of f-enthalpy, examples with higher f-enthalpy tend to have negative charge state, while those with lower enthalpy tend to have positive charge states

3. When the compounds are listed in increasing value of f-enthalpy, examples with lower fenthalpy (with labels "low" or "med") have either a substitution in Rh or a substitution in Zn. The first compound resulting from a vacancy with this ordering of f-enthalpy values occurs at index 29 (out of 52). Note that the converse is not true, that is, a substitution in Rh or a substitution in Zn does not result in a lower f-enthalpy.

4. Equivalently, if the impurity is a vacancy in $\mathrm{Rh}$, or a vacancy in $\mathrm{Zn}$, or a vacancy in $\mathrm{O}$, then the f-enthalpy is "med" or "high". The converse is not true as "med" or "high" f-enthalpy can result from substitution impurities.

5. There are three compounds with negative f-enthalpy, all resulting from a Cr substitution on $\mathrm{Rh}$ or $\mathrm{Zn}$.

6. The following observation made for $\mathrm{Co} 2 \mathrm{ZnO} 4$, does not hold good for $\mathrm{Rh} 2 \mathrm{ZnO} 4$ : "For a given species which substitutes a $\mathrm{Zn}$ atom, the ones with lower charge state have a higher enthalpy (i.e., if we take the compounds where species $\mathrm{X}$ replaces a $\mathrm{Zn}$ atom and arrange them by decreasing charge state, the order would lead to increasing f-enthalpy values). However, this is not always the case when we consider species which substitute a Co atom." 


\begin{tabular}{|c|c|c|c|c|c|c|}
\hline Compound ID & impurity & PT row & PT col & charge state & f-enthalpy & label \\
\hline Mg_on_Rh_0 & $\mathrm{Mg}$ & 3 & 2 & 0 & 0.1236964375 & low \\
\hline Mg_on_Rh_-1 & $\mathrm{Mg}$ & 3 & 2 & -1 & 0.4948394375 & low \\
\hline Ca_on_Rh_0 & $\mathrm{Ca}$ & 4 & 2 & 0 & 0.5133934375 & low \\
\hline Ca_on_Rh_-1 & $\mathrm{Ca}$ & 4 & 2 & -1 & 1.1453564375 & low \\
\hline Sr_on_Rh_0 & $\mathrm{Sr}$ & 5 & 2 & 0 & 1.3901164375 & med \\
\hline Be_on_Rh_0 & $\mathrm{Be}$ & 2 & 2 & 0 & 1.5986374375 & med \\
\hline Be_on_Rh_-1 & $\mathrm{Be}$ & 2 & 2 & -1 & 1.7308044375 & med \\
\hline Sr_on_Rh_-1 & $\mathrm{Sr}$ & 5 & 2 & -1 & 2.0913034375 & med \\
\hline Ba_on_Rh_0 & $\mathrm{Ba}$ & 6 & 2 & 0 & 2.8719954375 & med \\
\hline Ba_on_Rh_-1 & $\mathrm{Ba}$ & 6 & 2 & -1 & 3.5874034375 & high \\
\hline
\end{tabular}

Table 5: Listed in increasing value of f-enthalpy, the compounds created from Rh2ZnO4 by substituting Rh with an element from column 2 of the periodic table (PT).

\begin{tabular}{|c|c|c|c|c|c|c|}
\hline Compound ID & impurity & PT row & PT col & charge state & f-enthalpy & label \\
\hline Mg_on_Zn_0 & $\mathrm{Mg}$ & 3 & 2 & 0 & 0.018697 & low \\
\hline Ca_on_Zn_0 & $\mathrm{Ca}$ & 4 & 2 & 0 & 0.306219 & low \\
\hline Sr_on_Zn_0 & $\mathrm{Sr}$ & 5 & 2 & 0 & 0.875269 & low \\
\hline Be_on_Zn_0 & $\mathrm{Be}$ & 2 & 2 & 0 & 1.110115 & low \\
\hline Ba_on_Zn_0 & $\mathrm{Ba}$ & 6 & 2 & 0 & 1.893281 & med \\
\hline
\end{tabular}

Table 6: Listed in increasing value of f-enthalpy, the compounds created from Rh2ZnO4 by substituting $Z n$ with an element from column 2 of the periodic table (PT).

7. When we consider compounds obtained by substituting Rh with an element from column 2 of the periodic table, we observe that the f-enthalpy increases as we go down the column of the periodic table (with the exception of Be which appears out of order) as shown in Table 5. Note that for a species, the lower charge state has higher f-enthalpy.

8. Similarly, when we consider compounds obtained by substituting $\mathrm{Zn}$ with an element from column 2 of the periodic table, we observe that the f-enthalpy increases as we go down the column of the periodic table (with the exception of Be which appears out of order) as shown in Table 6 . In this case, the only charge state is zero.

\subsection{Analysis of the $\mathrm{Mn} 2 \mathrm{CrO} 4$ periodic table dataset}

For $\mathrm{Mn} 2 \mathrm{CrO} 4$, the formation enthalpy values range from -2.08 to 12.36 . This dataset was obtained later in the project, and we decided not to split the data into three groups of "low", "medium" and "high" f-enthalpy values as we found that it did not contribute to the analysis. We make the following observations on this dataset: 
1. The substitutions for $\mathrm{Cr}$ and $\mathrm{Mn}$ tend to be from periodic table rows 4 or column 1 , though there are several exceptions to this.

2. The dataset has very few examples of positive charge states. Of the 49 samples, 25 are negative, 21 are zero, and only 3 have a positive charge state.

3. The more negative charge states tend to have higher formation enthalpy.

4. When the compounds are listed in increasing order of f-enthalpy, the lower f-enthalpy compounds tend to be a substitution on $\mathrm{Mn}$ or $\mathrm{Cr}$. The first compound resulting from a vacancy in this ordering of f-enthalpy values occurs at index 21 (out of 49). Note that the converse is not true, that is, a substitution in $\mathrm{Cr}$ or a substitution in $\mathrm{Mn}$ does not result in a lower f-enthalpy value.

5. The three lowest f-enthalpy values (two negative, one near zero) are for $\mathrm{Mg}$ substitutions on $\mathrm{Cr}$ or Mn.

6. Of the six highest f-enthalpy values, five involve substitutions with Na. These are all the compounds in this dataset that were generated using a substitution with Na.

7. It is curious that the highest and lowest f-enthalpy for this spinel material result from substitutions involving elements from row 3 of the periodic table (columns 1 and 2).

8. For a substitution, the lower charge states (negative is lower than zero, which is lower than positive), have higher f-enthalpy, regardless of which species ( $\mathrm{Cr}$ or $\mathrm{Mn}$ ) is being substituted.

\subsection{Summary of the analysis of periodic table datasets}

The analysis of the periodic table datasets for the three spinel materials - Co2ZnO4, $\mathrm{Rh} 2 \mathrm{ZnO} 4$, and $\mathrm{Mn} 2 \mathrm{CrO} 4$ - indicates that there are some similarities. First, vacancies tend to result in higher formation enthalpy than substitutions. Second, for substitutions, the lower charge states have higher formation enthalpy values.

Our analysis also indicated that $\mathrm{Co} 2 \mathrm{ZnO} 4$ and $\mathrm{Rh} 2 \mathrm{ZnO} 4$ were more similar to each other than to $\mathrm{Mn} 2 \mathrm{CrO}$. However, given the small sample sizes of the compounds generated from the three spinel materials, and the resulting biases in sampling the design space, we need to be careful in extrapolating any of the observations made for these three materials to other spinel materials.

\section{Analysis of the properties data}

In this dataset, instead of the location of the impurity in the periodic table, we consider the properties associated with the atomic species that form the compound derived from the spinel material through either a vacancy or a substitution of one of the species.. The intent is to determine if the values of the properties are reflective of the formation enthalpy.

The first task in the analysis is to derive an appropriate representation for the properties of each compound. However, there are several challenges to this. First, not all properties are available for all atomic species. Second, we need a representation that treats vacancies and 
substitutions similarly. This is difficult because in the case of a vacancy, we still have three atomic species representing the compound, while in the case of a substitution, there is a fourth atomic species. We not only need to include the properties of this fourth species, but also indicate which species it is replacing. One approach is to consider a representation where the properties of each of the three atomic species are concatenated into a feature vector to describe the compound. A vacancy or a substitution is then represented by appropriately changing the properties of the species being "vacated" or "substituted".

We explored two ways in which we can incorporate this change, and suitably represent the properties of the three species (denoted by "A", "B", and "C", where "C" represents oxygen):

- Using differences: This is similar to the case of ratios, except we take the differences. If there is no vacancy or substitution for one of the three species, A, B, or C, we assign a property its value, if it is available; and $\mathrm{N} / \mathrm{A}$, if it is not. If a species has a vacancy, we assign the property a value of 0.0 , if it is available; and $\mathrm{N} / \mathrm{A}$, if it is not. If a species has a substitution, we take the difference of the values of the property for the species and the impurity, assuming both are available; and N/A, if one or both values are unavailable. Having generated the properties for each compound derived from a spinel material (such as $\mathrm{Co} 2 \mathrm{ZnO} 4)$, we then remove any properties assigned a value of $\mathrm{N} / \mathrm{A}$.

- Using ratios: In this case, if there is no vacancy or substitution for one of the three species, A, B, or C, we assign a value of 1.0 to a property, if it is available; and N/A, if it is not. If a species has a vacancy, we assign the property a value of 0.0 , if it is available; and $\mathrm{N} / \mathrm{A}$, if it is not. If a species has a substitution, we take the ratio of the property of the impurity to the property of the species, assuming both are available; and N/A, if one or both are unavailable. Having generated the properties for each compound derived from a spinel material (such as $\mathrm{Co} 2 \mathrm{ZnO} 4$ ), we then remove any properties assigned a value of $\mathrm{N} / \mathrm{A}$.

Note that missing properties are not included in creating the dataset resulting in fewer columns and different properties for each of the three atomic species and their impurity. It may also mean that the properties used to represent different spinel materials might be different.

We also observe that any approach to using the properties of the atomic species, A, B, and $\mathrm{C}$, to represent a compound also implies that we will have cases where two compounds have the same representation for the properties and differ only in the charge state and the f-enthalpy values. Since there appears to be no direct correlation between the charge state and the f-enthalpy, this observation indicates that we might be missing some information that is relevant to predicting the formation enthalpy of a compound.

Our early experiments with the two representations - one based on ratios and the other on differences - using the data for the $\mathrm{Co} 2 \mathrm{ZnO} 4$ spinel indicated that there were several issues with the use of differences in the representation of a compound. First, the use of differences could result in negative values for some properties. Second, there can be a large variation in the values of a property. For example, the atomization property for Co has value 426000, which is the value used in the dataset if there is no substitution or vacancy on Co. However, a Co vacancy is represented with a 0.0 value, and a substitution could result in a value as low as -89000. Thus, the use of differences can introduce an artificially large variation in the values of a property. A similar observation can be made for properties such as thermal expansion, 
which has a value of $1.3 \mathrm{e}-05$ for Co. Setting the feature value corresponding to this property to 0.0 in case of a Co vacancy artificially introduces a large variation as all other values for this feature are near 1.0e-05. And finally, since the properties of an atomic species differ in value (e.g., the 426000 for atomization versus 1.3e-05 for thermal expansion), we need to normalize each property prior to the analysis. The artificial variation introduced by the use of differences could adversely affect this normalization.

In light of these observations, we consider only the dataset generated using the ratios of properties in our analysis. We refer to these ratios as "features" representing each compound.

Having generated the properties dataset for the three spinel materials, we found there were still challenges to the analysis:

- There are relatively few samples for analysis for each spinel, with around 50 compounds generated for each of $\mathrm{Co} 2 \mathrm{ZnO} 4, \mathrm{Rh} 2 \mathrm{ZnO} 4$, and $\mathrm{Mn} 2 \mathrm{Cr} 04$. This makes it difficult to draw strong conclusions as they may reflect the choice of samples rather than any scientific insights.

- Not all properties are available for all atomic species. If a property is unavailable for even one compound in the dataset, it is removed from consideration. This may result in the removal of features that are relevant to the determination of the formation enthalpy.

- As explained earlier, for each spinel material, any impurity that appears in more than one charge state results in compounds that differ in their representation only in the charge state and formation enthalpy; all other features are identical. As there does not appear to be a correlation between the charge state and the formation enthalpy, this indicates that some information necessary for predicting the f-enthalpy is unavailable in our analysis.

- Since there are few sample compounds for each spinel material, it is unclear if the design space has been adequately sampled.

- Several of the features (the ratios of the properties) are correlated. These are removed prior to analysis (see the results for each spinel material for more details).

Given these issues, we were unable to gain any insights into the data using traditional data analysis techniques such as feature selection or regression.

We next wanted to determine if we could learn something from the properties datasets. For example, for each spinel material, if we considered the compounds with the same value of the charge state, would compounds which were near each other in the feature space have similar formation enthalpy values? If this were the case, it would indicate that, for a given charge state, the representation of the compounds is predictive of the formation enthalpy. Note that the converse is not necessarily true as the design space of compounds is not well sampled. For example, two nearby compounds may have quite different values for formation enthalpy if they belong to two groups which are near each other in feature space, but have very different f-enthalpy values. Or, two groups farther away in feature space, could have very similar fenthalpy values. However, if there is no correlation between the f-enthalpy of a compound and those of (at least) some of its nearest neighbors, then it is unlikely that the representation we have chosen is an appropriate one. We next describe the results of these analysis for the properties data for the three spinel materials. 


\begin{tabular}{|l|l|}
\hline Feature to keep & Correlated features to remove \\
\hline ARsingle_bond_radius & $\begin{array}{l}\text { ARmolar_volume, ARcovalent_radius, ARdouble_bond_radius, } \\
\text { ARatomic_radius, AR_orbital_radius_s, ARorbital_radius_o }\end{array}$ \\
\hline ARpauling & ARsanderson, ARallred_rochow, ARpettifor, ARfirst_ionization_energy \\
\hline ARatomization & ARmelting_point, ARvaporization, ARfusion, ARboiling_point \\
\hline ARatomic_weight & ARatomic_number \\
\hline BRsingle_bond_radius & $\begin{array}{l}\text { BRmolar_volume, BRcovalent_radius, BRdouble_bond_radius, } \\
\text { BRatomic_radius, BR_orbital_radius_s, BRorbital_radius_o }\end{array}$ \\
\hline BRpauling & BRsanderson, BRallred_rochow, BRpettifor, BRfirst_ionization_energy \\
\hline BRatomization & BRmelting_point, BRvaporization, BRfusion, BRboiling_point \\
\hline BRatomic_weight & BRatomic_number \\
\hline
\end{tabular}

Table 7: The features to remove because they are correlated to other features in the Co2ZnO4 dataset. The $A R$ and $B R$ prefix indicate the ratios of the property for species $A$ and its impurity and species $B$ and its impurity, respectively.

\begin{tabular}{|l|l|l|l|}
\hline ARsingle_bond_radius & ARpauling & ARatomization & ARatomic_weight \\
\hline ARthermal_conductivity & ARrigidity_modulus & ARdensity & ARthermal_expansion \\
\hline BRsingle_bond_radius & BRpauling & BRatomization & BRatomic_weight \\
\hline BRthermal_conductivity & BRrigidity_modulus & BRdensity & BRthermal_expansion \\
\hline CRatomic_weight & cstate & & \\
\hline
\end{tabular}

Table 8: The uncorrelated features used in the analysis of the properties data for Co2ZnO4. The AR and $B R$ prefix indicate the ratios of the property for species $A$ and its impurity and species $B$ and its impurity, respectively.

Caveat: These observations in the next three sections are based on a relatively small dataset (53 examples for $\mathrm{Co} 2 \mathrm{ZnO} 4,52$ for $\mathrm{Rh} 2 \mathrm{ZnO} 4$, and 49 for $\mathrm{Mn} 2 \mathrm{CrO} 4$ ). They are more qualitative in nature and may reflect the set of samples in the data rather than the physics. Therefore, any interpretation and extrapolation must be done with care.

\subsection{Analysis of the $\mathrm{Co} 2 \mathrm{ZnO} 4$ properties dataset}

For $\mathrm{Co} 2 \mathrm{ZnO} 4$, we first identified the features that were correlated to the others and removed them from consideration. Table 7 lists the features which are correlated; only one of these is considered in the analysis. Table 8 lists the features that remain after the removal of the correlated ones.

The Co2ZnO4 dataset has 21 compounds with charge state 0 and 14 with charge state -1 ; the remaining charge states have very few compounds and are not considered in the analysis. Once the correlated features have been removed, we consider each of the charge states separately in our analysis to avoid the issue discussed in the previous section.

In Appendix A, for charge states 0 and -1 , we present the distance between each compound and its nearest and farthest neighbors, as well as the nearest two compounds, their distances, 
and the corresponding formation enthalpy values for these compounds. All distances considered are Euclidean distances.

An analysis of Tables 12 and 14 indicates that some compounds have nearest neighbors that are quite close, while others have their nearest neighbors much farther away. This may indicate possible locations where additional samples might be added.

The analysis of Tables 13 and 15 is somewhat inconclusive as it is unclear if the nearest two neighbors of a compound have formation enthalpies which are close to the formation enthalpy of the compound. However, we found that there is some pattern in the formation enthalpies of compounds with charge state 0 and charge state -1 that were generated using substitutions. Specifically, for some compounds, the difference in enthalpy values between the nearest neighbors with charge state 0 is very similar to the difference in enthalpy values between the nearest neighbors with charge state -1 . This may mean that given three of these values, we may be able to predict the fourth. Note that the distances between the nearest neighbors are calculated using only the features derived from the properties of the atomic species; the charge state is not included.

A closer examination of the $\mathrm{Co} 2 \mathrm{ZnO} 4$ data indicated that the difference in formation enthalpy between two compounds in charge state 0 is often close to the difference in formation enthalpy between the same two compounds in charge state -1, regardless of whether the two compounds are nearest neighbors or not (that is, the distance between the features is not relevant). A similar statement can also be made about the other charge states, though the number of samples is far fewer to rule out a chance coincidence.

In light of this observation, it might be worth looking into the compounds that do not exhibit this behavior to understand why this is the case.

\subsection{Analysis of the $\mathrm{Rh} 2 \mathrm{ZnO} 4$ properties dataset}

For $\mathrm{Rh} 2 \mathrm{ZnO} 4$, we also started by identifying the features that were correlated to the others and removed them from consideration. Table 9 lists the features which are correlated; only one of these is considered in the analysis. This list is slightly different from the correlated variables for $\mathrm{Co} 2 \mathrm{ZnO} 4$ as the sanderson variable was unavaiable and the orbital_radius_o was more closely related to the atomic_weight for the species A feature. The list of features that remain after the removal of the correlated ones are identical to those for $\mathrm{Co} 2 \mathrm{ZnO} 4$ (see Table 8).

The Rh2ZnO4 dataset has 21 compounds with charge state 0 and 14 with charge state -1 ; the remaining charge states have very few compounds and are not considered in the analysis. As with $\mathrm{Co} 2 \mathrm{ZnO} 4$, we removed the correlated features and analyzed the data for charge states 0 and -1 separately.

In Appendix B, for charge states 0 and -1, we present the distance between each compound and its nearest and farthest neighbors, as well as the nearest two compounds, their distances, and the corresponding formation enthalpy values for these compounds. All distances considered are Euclidean distances. The observations are very similar to those for $\mathrm{Co} 2 \mathrm{ZnO} 4$ and are repeated here for completeness.

Tables 17 and 19 indicate that some compounds have nearest neighbors that are quite close, while others have their nearest neighbors much farther away, indicating possible locations where additional samples might be added. 


\begin{tabular}{|l|l|}
\hline Feature to keep & Correlated features to remove \\
\hline ARsingle_bond_radius & $\begin{array}{l}\text { ARmolar_volume, ARcovalent_radius, ARdouble_bond_radius, } \\
\text { ARatomic_radius, AR_orbital_radius_s }\end{array}$ \\
\hline ARpauling & ARallred_rochow, ARpettifor, ARfirst_ionization_energy \\
\hline ARatomization & ARmelting_point, ARvaporization, ARfusion, ARboiling_point \\
\hline ARatomic_weight & ARatomic_number, AR_orbital_radius_o \\
\hline BRsingle_bond_radius & $\begin{array}{l}\text { BRmolar_volume, BRcovalent_radius, BRdouble_bond_radius, } \\
\text { BRatomic_radius, BR_orbital_radius_s, BRorbital_radius_o }\end{array}$ \\
\hline BRpauling & BRallred_rochow, BRpettifor, BRfirst_ionization_energy \\
\hline BRatomization & BRmelting_point, BRvaporization, BRfusion, BRboiling_point \\
\hline BRatomic_weight & BRatomic_number \\
\hline
\end{tabular}

Table 9: The features to remove because they are correlated to other features in the Rh2ZnO4 dataset. The $A R$ and BR prefix indicate the ratios of the property for species $A$ and its impurity and species $B$ and its impurity, respectively.

The analysis of Tables 18 and 20 is somewhat inconclusive as it is unclear if the nearest two neighbors of a compound have similar values of formation enthalpies. However, the pattern we had observed in $\mathrm{Co} 2 \mathrm{ZnO} 4$ in the formation enthalpies of compounds with charge states 0 and -1 persists for $\mathrm{Rh} 2 \mathrm{ZnO} 4$. We found that the difference in formation enthalpy between two compounds in charge state 0 is often close to the difference in formation enthalpy between the same two compounds in charge state -1 , regardless of whether the two compounds are nearest neighbors or not (that is, the distance between the features is not relevant). A similar statement can also be made about the other charge states, though the number of samples is far fewer to rule out a chance coincidence. As with $\mathrm{Co} 2 \mathrm{ZnO} 4$, it might be worth looking into the compounds that do not exhibit this behavior to understand why this is the case.

\subsection{Analysis of the $\mathrm{Mn} 2 \mathrm{CrO} 4$ properties dataset}

For the $\mathrm{Mn} 2 \mathrm{CrO} 4$ dataset, we found that there was a greater correlation between the features correlated to $\mathrm{AR}(\mathrm{BR})$ atomization and $\mathrm{AR}(\mathrm{BR})$ pauling than observed in the Co2ZnO4 or $\mathrm{Rh} 2 \mathrm{ZnO} 4$ data. In Table 10, the features to remove are assigned to the most closely correlated feature to keep. The uncorrelated features used in the analysis are listed in Table 11; note that these are a different set from $\mathrm{Co} 2 \mathrm{ZnO} 4$ and $\mathrm{Rh} 2 \mathrm{ZnO} 4$ due to the properties available for the atomic species and the features identified as correlated.

The $\mathrm{Mn} 2 \mathrm{CrO} 4$ dataset has 21 compounds with charge state 0 and 17 with charge state -1 ; the remaining charge states have very few compounds and are not considered in the analysis. As before, we remove the correlated features and analyze charge states 0 and -1 separately.

In Appendix C, for charge states 0 and -1 , we present the distance between each compound and its nearest and farthest neighbors, as well as the nearest two compounds, their distances, and the corresponding formation enthalpy values for these compounds. All distances considered are Euclidean distances. Our analysis results are very similar to those of the other two compounds and not repeated here. 


\begin{tabular}{|l|l|}
\hline Feature to keep & Correlated features to remove \\
\hline ARsingle_bond_radius & $\begin{array}{l}\text { ARmolar_volume, ARcovalent_radius, ARdouble_bond_radius, } \\
\text { ARatomic_radius, AR_orbital_radius_s }\end{array}$ \\
\hline ARpauling & $\begin{array}{l}\text { ARallred_rochow, ARpettifor, ARfirst_ionization_energy, AR- } \\
\text { bulk_modulus }\end{array}$ \\
\hline ARatomization & $\begin{array}{l}\text { ARsanderson, ARmelting_point, ARvaporization, ARfusion, ARboil- } \\
\text { ing_point }\end{array}$ \\
\hline ARatomic_weight & ARatomic_number \\
\hline BRsingle_bond_radius & $\begin{array}{l}\text { BRmolar_volume, BRcovalent_radius, } \\
\text { BRatomic_radius, BR_orbital_radius_s, BRorbital_radius_o }\end{array}$ \\
\hline BRpauling & $\begin{array}{l}\text { BRallred_rochow, BRpettifor, BRfirst_ionization_energy, } \\
\text { bulk_modulus }\end{array}$ \\
\hline BRatomization & $\begin{array}{l}\text { BRsanderson, BRmelting_point, BRvaporization, BRfusion, BRboil- } \\
\text { ing_point }\end{array}$ \\
\hline BRatomic_weight & BRatomic_number \\
\hline
\end{tabular}

Table 10: The features to remove because they are correlated to other features in the $\mathrm{Mn}_{2} \mathrm{CrO} 4$ dataset. The $A R$ and BR prefix indicate the ratios of the property for species $A$ and its impurity and species $B$ and its impurity, respectively.

\begin{tabular}{|l|l|l|l|}
\hline ARsingle_bond_radius & ARpauling & ARatomization & ARsound_velocity \\
\hline ARatomic_weight & ARthermal_conductivity & ARdensity & AR_orbital_radius_o \\
\hline BRsingle_bond_radius & BRpauling & BRatomization & BRsound_velocity \\
\hline BRatomic_weight & BRthermal_conductivity & BRdensity & BR_orbital_radius_o \\
\hline CRatomic_weight & cstate & & \\
\hline
\end{tabular}

Table 11: The uncorrelated features used in the analysis of the properties data for Mn2CrO4. The AR and $B R$ prefix indicate the ratios of the property for species $A$ and its impurity and species $B$ and its impurity, respectively.

\subsection{Summary of analysis of properties dataset}

The analysis of the properties dataset for the three spinel materials is challenging, especially as it is unclear what is the best way to represent a compound in terms of the properties of its constituent atomic species; the sample size is quite small and the design space not adequately sampled; not all properties are available for all atomic species; and we need to represent compounds that differ only in the charge state, but may have very different formation enthalpy values. All this indicates that some information relevant to predicting the formation enthalpy may be missing. The crystal structure of a compound may play a role here, though converting the locations of the different atoms in 3-D space into relevant features is an open question.

We considered two representations of the properties - one based on differences and the other on ratios between a species and the impurity. We analyzed the dataset generated using the ratios as it was better behaved.

Our attempts to find correlations between the features describing a compound and its formation enthalpy were inconclusive. We did find that, if we consider the compounds generated 
using a substitution, the difference between the formation enthalpy of two compounds with charge state 0 appears to be close to the difference between the formation enthalpy of the corresponding compounds in charge state -1 . This may make it possible to predict one given the other three. The observation extends to other charge states as well, though there are too few examples to draw a conclusion. However, the observation is not universally true and it may be worth investigating the compounds for which this is the case. In addition, the distance to the nearest neighbor for each compound could be used to add additional sample points as appropriate, resulting in a more complete coverage of the design space.

\section{Conclusions and future work}

In this report, we discussed the analysis of a data set of compounds derived from threee different spinel materials with the intent of determining if it was possible to predict the formation enthalpy of the compounds. There were several challenges to the analysis, including the small number of compounds available for each spinel, the determination of an appropriate representation of a compound in terms of the characteristics of its constituent atomic species, the suitable incorporation of the charge state in the analysis, and the need to treat impurities and vacancies similarly.

Our analysis indicated that traditional data mining techniques, such as feature selection and regression were not much help in the analysis. We obtained some qualitative insights into the compounds and the prediction of the formation enthalpy by considering two datasets - one based on the periodic table and the other based on atomic properties. The analysis of the latter indicated what appears to be a pattern in the differences in formation enthalpy between two compounds with charge state 0 and the same compounds with charge state -1 . This may extend to other charge states, though the we did not have enough samples to validate this observation.

Based on the analysis, an obvious next step would be to analyze further the observation on the differences in formation enthalpy between two compounds with different charge states. Increasing the size of the datasets and seeing how many of the qualitative observations remain valid could be another venue of exploration. And finally, there remains the need to identify an appropriate representation for a compound that adequately reflects the formation enthalpy.

\section{Acknowledgment}

This work was performed as part of the ARRA-funded DOE SciDAC-e project, MINDES - Data Mining for Inverse Design, in support of the Center for Inverse Design EFRC. The analysis was done in collaboration with Mayeul d'Avezac from NREL, who provided the data and the domain expertise. The idea of using ratios of properties was motivated by a suggestion by Haowei Peng of NREL who proposed using the differences of properties. The analysis of $\mathrm{Co} 2 \mathrm{ZnO} 4$ and $\mathrm{Rh} 2 \mathrm{ZnO} 4$ was funded by the SciDAC-e program; the analysis of $\mathrm{Mn} 2 \mathrm{CrO} 4$ was unfunded work. I appreciate the support and interest of Mayeul d'Avezac, as well as the EFRC Directors, William Tumas and Alex Zunger.

LLNL-TR-582974: This work performed under the auspices of the U.S. Department of Energy by Lawrence Livermore National Laboratory under Contract DE-AC52-07NA27344. 


\section{References}

[1] Center for Inverse Design web page, 2012. http://www . centerforinversedesign.org/.

[2] Fang, K.-T., Li, R., And Sudjinnto, A. Design and Modeling for Computer Experiments. Chapman and Hall/CRC Press, Boca Raton, FL, 2005.

[3] MINDES: Data Mining for Inverse Design Project web page, 2012. https://computation . llnl.gov/casc/StarSapphire/MINDES.html. 
A Results for the analysis of properties data for $\mathrm{Co} 2 \mathrm{ZnO} 4$ 


\begin{tabular}{|c|c|c|c|c|}
\hline \multirow[t]{2}{*}{ Compound ID } & \multicolumn{2}{|l|}{ nearest neighbor } & \multicolumn{2}{|c|}{ farthest neighbor } \\
\hline & compound & distance & compound & distance \\
\hline Co2Zn_Zn_vacancy_0 & Co2ZnO4_O_vacancy_0 & 3 & Co2ZnO4_Ba_on_Co_0 & 15.4745 \\
\hline Co2ZnO4_Co_vacancy_0 & Co2ZnO4_O_vacancy_0 & 3 & Co2ZnO4_Ba_on_Co_0 & 16.8052 \\
\hline Co2ZnO4_O_vacancy_0 & Co2ZnO4_Co_on_Zn_0 & 1.87673 & Co2ZnO4_Ba_on_Co_0 & 15.2467 \\
\hline Co2ZnO4_Zn_on_Co_0 & Co2ZnO4_O_vacancy_0 & 2.65904 & Co2ZnO4_Ba_on_Co_0 & 14.4937 \\
\hline Co2ZnO4_Co_on_Zn_0 & Co2ZnO4_O_vacancy_0 & 1.87673 & Co2ZnO4_Ba_on_Co_0 & 15.2965 \\
\hline Co2ZnO4_Be_on_Zn_0 & Co2ZnO4_Mg_on_Zn_0 & 5.2123 & Co2ZnO4_Ba_on_Co_0 & 16.8093 \\
\hline Co2ZnO4_Be_on_Co_0 & Co2ZnO4_Mg_on_Co_0 & 5.89104 & Co2ZnO4_Ba_on_Co_0 & 16.9548 \\
\hline Co2ZnO4_Mg_on_Zn_0 & Co2ZnO4_Ca_on_Zn_0 & 4.21729 & Co2ZnO4_Ba_on_Co_0 & 15.9273 \\
\hline Co2ZnO4_Mg_on_Co_0 & Co2ZnO4_Zn_on_Co_0 & 5.10328 & Co2ZnO4_Ba_on_Co_0 & 12.4667 \\
\hline Co2ZnO4_Ca_on_Zn_0 & Co2ZnO4_Sr_on_Zn_0 & 3.72967 & Co2ZnO4_Ba_on_Co_0 & 16.3977 \\
\hline Co2ZnO4_Ca_on_Co_0 & Co2ZnO4_Sr_on_Co_0 & 4.05854 & Co2ZnO4_Ba_on_Zn_0 & 14.301 \\
\hline Co2ZnO4_Sr_on_Zn_0 & Co2ZnO4_Ca_on_Zn_0 & 3.72967 & Co2ZnO4_Ba_on_Co_0 & 16.667 \\
\hline Co2ZnO4_Sr_on_Co_0 & Co2ZnO4_Ca_ & 5854 & Co2ZnO4_Ba_on_Zn_0 & 15.3546 \\
\hline Co2ZnO4_Ba_on_Zn_0 & Co2ZnO4_Sr_on_Zn_0 & 3.74677 & Co2ZnO4_Ba_on_Co_0 & 18.0709 \\
\hline Co2ZnO4_Ba_on_Co_0 & Co2ZnO4_Sr_on_Co_0 & 4.14905 & Co2ZnO4_Ba_on_Zn_0 & 18.0709 \\
\hline Co2ZnO4_Ti_on_Zn_0 & Co2ZnO4_V_on_Zn_0 & 1.64389 & Co2ZnO4_Ba_on_Co_0 & 16.082 \\
\hline Co2ZnO4_Ti_on_Co_0 & Co2ZnO4_V_on_Co_0 & 1.428 & Co2ZnO4_Ba_on_Co_0 & 13.8086 \\
\hline Co2ZnO4_V_on_Zn_0 & Co2ZnO4_Ti_on_Zn_0 & 1.64389 & Co2ZnO4_Ba_on_Co_0 & 15.7202 \\
\hline Co2ZnO4_V_on_Co_0 & Co2ZnO4_Ti_on_Co_0 & 1.428 & Co2ZnO4_Ba_on_Co_0 & 14.1144 \\
\hline Co2ZnO4_Cr_on_Zn_0 & Co2ZnO4_V_on_Zn_0 & 3.69606 & Co2ZnO4_Ba_on_Co_0 & 16.0967 \\
\hline Co2ZnO4_Cr_on_Co_0 & Co2ZnO4_O_vacancy_0 & 1.9918 & Co2ZnO4_Ba_on_Co_0 & 15.6155 \\
\hline
\end{tabular}

Table 12: The distances to the nearest and farthest neighbors for the 21 compounds, with charge state 0, obtained from $\mathrm{Co}_{\mathrm{ZnO} O 4}$. 


\begin{tabular}{|c|c|c|c|c|}
\hline \multirow[t]{2}{*}{ Compound ID } & \multirow[t]{2}{*}{ f-enthalpy } & \multicolumn{3}{|c|}{ top two nearest neighbors } \\
\hline & & compound & distance & f-enthalpy \\
\hline \multirow[t]{2}{*}{ Co2ZnO4_Zn_vacancy_0 } & 2.34246 & Co2ZnO4_O_vacancy_0 & 3 & 3.22481 \\
\hline & & Co2ZnO4_Cr_on_Co_0 & 3.31169 & -0.081488 \\
\hline \multirow[t]{2}{*}{ Co2ZnO4_Co_vacancy_0 } & 2.89641 & Co2ZnO4_O_vacancy_0 & 3 & 3.22481 \\
\hline & & Co2ZnO4_Co_on_Zn_0 & 3.24378 & 0.273712 \\
\hline \multirow[t]{2}{*}{ Co2ZnO4_O_vacancy_0 } & 3.22481 & Co2ZnO4_Co_on_Zn_0 & 1.87673 & 0.273712 \\
\hline & & Co2ZnO4_Cr_on_Co_0 & 1.9918 & -0.081488 \\
\hline \multirow[t]{2}{*}{ Co2ZnO4_Zn_on_Co_0 } & 1.04821 & Co2ZnO4_O_vacancy_0 & 2.65904 & 3.22481 \\
\hline & & Co2ZnO4_Co_on_Zn_0 & 2.93132 & 0.273712 \\
\hline \multirow[t]{2}{*}{ Co2ZnO4_Co_on_Zn_0 } & 0.273712 & Co2ZnO4_O_vacancy_0 & 1.87673 & 3.22481 \\
\hline & & Co2ZnO4_Cr_on_Co_0 & 2.34295 & -0.081488 \\
\hline \multirow[t]{2}{*}{ Co2ZnO4_Be_on_Zn_0 } & 0.416459 & Co2ZnO4_Mg_on_Zn_0 & 5.2123 & 0.10936 \\
\hline & & Co2ZnO4_Co_on_Zn_0 & 6.90585 & 0.273712 \\
\hline \multirow[t]{2}{*}{ Co2ZnO4_Be_on_Co_0 } & 1.21764 & Co2ZnO4_Mg_on_Co_0 & 5.89104 & 0.700634 \\
\hline & & Co2ZnO4_Cr_on_Co_0 & 6.67931 & -0.081488 \\
\hline \multirow[t]{2}{*}{ Co2ZnO4_Mg_on_Zn_0 } & 0.10936 & Co2ZnO4_Ca_on_Zn_0 & 4.21729 & 1.01186 \\
\hline & & Co2ZnO4_Co_on_Zn_0 & 4.45475 & 0.273712 \\
\hline \multirow[t]{2}{*}{ Co2ZnO4_Mg_on_Co_0 } & 0.700634 & Co2ZnO4_Zn_on_Co_0 & 5.10328 & 1.04821 \\
\hline & & Co2ZnO4_V_on_Co_0 & 5.8464 & 1.0342 \\
\hline \multirow[t]{2}{*}{ Co2ZnO4_Ca_on_Zn_0 } & 1.01186 & Co2ZnO4_Sr_on_Zn_0 & 3.72967 & 1.98969 \\
\hline & & Co2ZnO4_Mg_on_Zn_0 & 4.21729 & 0.10936 \\
\hline \multirow[t]{2}{*}{ Co2ZnO4_Ca_on_Co_0 } & 1.88383 & Co2ZnO4_Sr_on_Co_0 & 4.05854 & 3.26647 \\
\hline & & Co2ZnO4_Mg_on_Co_0 & 5.96399 & 0.700634 \\
\hline \multirow[t]{2}{*}{ Co2ZnO4_Sr_on_Zn_0 } & 1.98969 & Co2ZnO4_Ca_on_Zn_0 & 3.72967 & 1.01186 \\
\hline & & Co2ZnO4_Ba_on_Zn_0 & 3.74677 & 3.52111 \\
\hline \multirow[t]{2}{*}{ Co2ZnO4_Sr_on_Co_0 } & 3.26647 & Co2ZnO4_Ca_on_Co_0 & 4.05854 & 1.88383 \\
\hline & & Co2ZnO4_Ba_on_Co_0 & 4.14905 & 5.33446 \\
\hline \multirow[t]{2}{*}{ Co2ZnO4_Ba_on_Zn_0 } & 3.52111 & Co2ZnO4_Sr_on_Zn_0 & 3.74677 & 1.98969 \\
\hline & & Co2ZnO4_Ca_on_Zn_0 & 7.32168 & 1.01186 \\
\hline \multirow[t]{2}{*}{ Co2ZnO4_Ba_on_Co_0 } & 5.33446 & Co2ZnO4_Sr_on_Co_0 & 4.14905 & 3.26647 \\
\hline & & Co2ZnO4_Ca_on_Co_0 & 7.98301 & 1.88383 \\
\hline \multirow[t]{2}{*}{ Co2ZnO4_Ti_on_Zn_0 } & 3.70883 & Co2ZnO4_V_on_Zn_0 & 1.64389 & 2.91725 \\
\hline & & Co2ZnO4_Co_on_Zn_0 & 4.52005 & 0.273712 \\
\hline \multirow[t]{2}{*}{ Co2ZnO4_Ti_on_Co_0 } & 1.74786 & Co2ZnO4_V_on_Co_0 & 1.428 & 1.0342 \\
\hline & & Co2ZnO4_Cr_on_Co_0 & 3.88805 & -0.081488 \\
\hline \multirow[t]{2}{*}{ Co2ZnO4_V_on_Zn_0 } & 2.91725 & Co2ZnO4_Ti_on_Zn_0 & 1.64389 & 3.70883 \\
\hline & & Co2ZnOH_Co_on_Zn_0 & 3.01461 & 0.273712 \\
\hline \multirow[t]{2}{*}{ Co2ZnO4_V_on_Co_0 } & 1.0342 & Co2ZnO4_Ti_on_Co_0 & 1.428 & 1.74786 \\
\hline & & Co2ZnO4_Cr_on_Co_0 & 2.62654 & -0.081488 \\
\hline \multirow[t]{2}{*}{ Co2ZnO4_Cr_on_Zn_0 } & 2.32195 & Co2ZnO4_V_on_Zn_0 & 3.69606 & 2.91725 \\
\hline & & Co2ZnOO4_Co_on_Zn_0 & 3.85706 & 0.273712 \\
\hline \multirow[t]{2}{*}{ Co2ZnO4_Cr_on_Co_0 } & -0.081488 & Co2ZnO4_O_vacancy_0 & 1.9918 & 3.22481 \\
\hline & & Co2ZnO4_Co_on_Zn_0 & 2.34295 & 0.273712 \\
\hline
\end{tabular}

Table 13: The distances to the nearest two neighbors, and their f-enthalpy values, for the 21 compounds, with charge state 0, obtained from Co2ZnO4. 


\begin{tabular}{|c|c|c|c|c|}
\hline \multirow{2}{*}{ Compound ID } & \multicolumn{2}{|c|}{ nearest neighbor } & \multicolumn{2}{c|}{ farthest neighbor } \\
\cline { 2 - 5 } & compound & distance & compound & distance \\
\hline Co2ZnO4_Zn_vacancy_-1 & Co2ZnO4_Cr_on_Co_-1 & 3.31169 & Co2ZnO4_Ba_on_Co_-1 & 15.4745 \\
\hline Co2ZnO4_Co_vacancy_-1 & Co2ZnO4_Cr_on_Co_-1 & 3.83354 & Co2ZnO4_Ba_on_Co_-1 & 16.8052 \\
\hline Co2ZnO4_Zn_on_Co_-1 & Co2ZnO4_Cr_on_Co_-1 & 3.31479 & Co2ZnO4_Ba_on_Co_-1 & 14.4937 \\
\hline Co2ZnO4_Be_on_Co_-1 & Co2ZnO4_Mg_on_Co_-1 & 5.89104 & Co2ZnO4_Ba_on_Co_-1 & 16.9548 \\
\hline Co2ZnO4_Mg_on_Co_-1 & Co2ZnO4_Zn_on_Co_-1 & 5.10328 & Co2ZnO4_Ba_on_Co_-1 & 12.4667 \\
\hline Co2ZnO4_Ca_on_Co_-1 & Co2ZnO4_Sr_on_Co_-1 & 4.05854 & Co2ZnO4_Co_vacancy_-1 & 12.1707 \\
\hline Co2ZnO4_Sr_on_Co_-1 & Co2ZnO4_Ca_on_Co_-1 & 4.05854 & Co2ZnO4_Co_vacancy_-1 & 13.5127 \\
\hline Co2ZnO4_Ba_on_Co_-1 & Co2ZnO4_Sr_on_Co_-1 & 4.14905 & Co2ZnO4_Be_on_Co_-1 & 16.9548 \\
\hline Co2ZnO4_Ti_on_Zn_-1 & Co2ZnO4_V_on_Zn_-1 & 1.64389 & Co2ZnO4_Ba_on_Co_-1 & 16.082 \\
\hline Co2ZnO4_Ti_on_Co_-1 & Co2ZnO4_V_on_Co_-1 & 1.428 & Co2ZnO4_Ba_on_Co_-1 & 13.8086 \\
\hline Co2ZnO4_V_on_Zn_-1 & Co2ZnO4_Ti_on_Zn_-1 & 1.64389 & Co2ZnO4_Ba_on_Co_-1 & 15.7202 \\
\hline Co2ZnO4_V_on_Co_-1 & Co2ZnO4_Ti_on_Co_-1 & 1.428 & Co2ZnO4_Ba_on_Co_-1 & 14.1144 \\
\hline Co2ZnO4_Cr_on_Zn_-1 & Co2ZnO4_V_on_Zn_-1 & 3.69606 & Co2ZnO4_Ba_on_Co_-1 & 16.0967 \\
\hline Co2ZnO4_Cr_on_Co_-1 & Co2ZnO4_V_on_Co_-1 & 2.62654 & Co2ZnO4_Ba_on_Co_-1 & 15.6155 \\
\hline
\end{tabular}

Table 14: The distances to the nearest and farthest neighbors for the 14 compounds, with charge state -1, obtained from Co2ZnO4. 


\begin{tabular}{|c|c|c|c|c|}
\hline \multirow[t]{2}{*}{ Compound ID } & \multirow[t]{2}{*}{ f-enthalpy } & \multicolumn{3}{|c|}{ top two nearest neighbors } \\
\hline & & compound & distance & f-enthalpy \\
\hline \multirow{2}{*}{ Co2ZnO4_Zn_vacancy_-1 } & \multirow[t]{2}{*}{2.73757} & Co2ZnO4_Cr_on_Co_-1 & 3.31169 & 2.0137 \\
\hline & & Co2ZnO4_V_on_Co_-1 & 3.73162 & 3.10042 \\
\hline \multirow{2}{*}{ Co2ZnO4_Co_vacancy_-1 } & \multirow[t]{2}{*}{3.58} & Co2ZnO4_Cr_on_Co_-1 & 3.83354 & 2.0137 \\
\hline & & Co2ZnO4_Zn_vacancy_-1 & 4 & 2.73757 \\
\hline \multirow{2}{*}{ Co2ZnO4_Zn_on_Co_-1 } & \multirow[t]{2}{*}{1.38399} & Co2ZnO4_Cr_on_Co_-1 & 3.31479 & 2.0137 \\
\hline & & Co2ZnO4_V_on_Co_-1 & 3.60493 & 3.10042 \\
\hline \multirow[t]{2}{*}{ Co2ZnO4_Be_on_Co_-1 } & \multirow[t]{2}{*}{1.32114} & Co2ZnO4_Mg_on_Co_-1 & 5.89104 & 1.00598 \\
\hline & & Co2ZnO4_Cr_on_Co_-1 & 6.67931 & 2.0137 \\
\hline \multirow[t]{2}{*}{ Co2ZnO4_Mg_on_Co_-1 } & \multirow[t]{2}{*}{1.00598} & Co2ZnO4_Zn_on_Co_-1 & 5.10328 & 1.38399 \\
\hline & & Co2ZnO4_V_on_Co_-1 & 5.8464 & 3.10042 \\
\hline \multirow[t]{2}{*}{ Co2ZnO4_Ca_on_Co_-1 } & \multirow[t]{2}{*}{2.47512} & Co2ZnO4_Sr_on_Co_-1 & 4.05854 & 3.95184 \\
\hline & & Co2ZnO4_Mg_on_Co_-1 & 5.96399 & 1.00598 \\
\hline \multirow[t]{2}{*}{ Co2ZnO4_Sr_on_Co_-1 } & \multirow[t]{2}{*}{3.95184} & Co2ZnO4_Ca_on_Co_-1 & 4.05854 & 2.47512 \\
\hline & & Co2ZnO4_Ba_on_Co_-1 & 4.14905 & 6.102 \\
\hline \multirow[t]{2}{*}{ Co2ZnO4_Ba_on_Co_-1 } & \multirow[t]{2}{*}{6.102} & Co2ZnO4_Sr_on_Co_-1 & 4.14905 & 3.95184 \\
\hline & & Co2ZnO4_Ca_on_Co_-1 & 7.98301 & 2.47512 \\
\hline \multirow[t]{2}{*}{ Co2ZnO4_Ti_on_Zn_-1 } & \multirow[t]{2}{*}{5.46123} & Co2ZnO4_V_on_Zn_-1 & 1.64389 & 4.83029 \\
\hline & & Co2ZnO4_Cr_on_Zn_-1 & 5.02217 & 4.07391 \\
\hline \multirow[t]{2}{*}{ Co2ZnO4_Ti_on_Co_-1 } & \multirow[t]{2}{*}{3.98017} & Co2ZnO4_V_on_Co_-1 & 1.428 & 3.10042 \\
\hline & & Co2ZnO4_Cr_on_Co_-1 & 3.88805 & 2.0137 \\
\hline \multirow[t]{2}{*}{ Co2ZnO4_V_on_Zn_-1 } & \multirow[t]{2}{*}{4.83029} & Co2ZnO4_Ti_on_Zn_-1 & 1.64389 & 5.46123 \\
\hline & & Co2ZnO4_Cr_on_Zn_-1 & 3.69606 & 4.07391 \\
\hline \multirow[t]{2}{*}{ Co2ZnO4_V_on_Co_-1 } & \multirow[t]{2}{*}{3.10042} & Co2ZnO4_Ti_on_Co_-1 & 1.428 & 3.98017 \\
\hline & & Co2ZnO4_Cr_on_Co_-1 & 2.62654 & 2.0137 \\
\hline \multirow[t]{2}{*}{ Co2ZnO4_Cr_on_Zn_-1 } & \multirow[t]{2}{*}{4.07391} & Co2ZnO4_V_on_Zn_-1 & 3.69606 & 4.83029 \\
\hline & & Co2ZnO4_Ti_on_Zn_-1 & 5.02217 & 5.46123 \\
\hline \multirow[t]{2}{*}{ Co2ZnO4_Cr_on_Co_-1 } & \multirow[t]{2}{*}{2.0137} & Co2ZnO4_V_on_Co_-1 & 2.62654 & 3.10042 \\
\hline & & Co2ZnO4_Zn_vacancy_-1 & 3.31169 & 2.73757 \\
\hline
\end{tabular}

Table 15: The distances to the nearest two neighbors, and their f-enthalpy values, for the 14 compounds, with charge state -1, obtained from $\mathrm{Co}_{2} \mathrm{ZnO} 4$. 


\begin{tabular}{|c|c|c|c|c|c|}
\hline \multirow{2}{*}{ Compound ID } & \multirow{2}{*}{ f-enthalpy } & \multicolumn{4}{|c|}{ nearest neighbor } \\
\cline { 3 - 6 } & & compound & distance & f-enthalpy & difference \\
\hline Co2ZnO4_Be_on_Co_0 & 1.21764 & Co2ZnO4_Mg_on_Co_0 & 5.89104 & 0.700634 & 0.517006 \\
Co2ZnO4_Be_on_Co_-1 & 1.32114 & Co2ZnO4_Mg_on_Co_-1 & 5.89104 & 1.00598 & 0.31516 \\
\hline Co2ZnO4_Mg_on_Co_0 & 0.700634 & Co2ZnO4_Zn_on_Co_0 & 5.10328 & 1.04821 & -0.347576 \\
Co2ZnO4_Mg_on_Co_-1 & 1.00598 & Co2ZnO4_Zn_on_Co_-1 & 5.10328 & 1.38399 & -0.37801 \\
\hline Co2ZnO4_Sr_on_Co_0 & 3.26647 & Co2ZnO4_Ca_on_Co_0 & 4.05854 & 1.88383 & 1.38264 \\
Co2ZnO4_Sr_on_Co_-1 & 3.95184 & Co2ZnO4_Ca_on_Co_-1 & 4.05854 & 2.47512 & 1.47672 \\
\hline Co2ZnO4_Ba_on_Co_0 & 5.33446 & Co2ZnO4_Sr_on_Co_0 & 4.14905 & 3.26647 & 2.06799 \\
Co2ZnO4_Ba_on_Co_-1 & 6.102 & Co2ZnO4_Sr_on_Co_-1 & 4.14905 & 3.95184 & 2.15016 \\
\hline Co2ZnO4_Ti_on_Zn_0 & 3.70883 & Co2ZnO4_V_on_Zn_0 & 1.64389 & 2.91725 & 0.79158 \\
Co2ZnO4_Ti_on_Zn_-1 & 5.46123 & Co2ZnO4_V_on_Zn_-1 & 1.64389 & 4.83029 & 0.63094 \\
\hline Co2ZnO4_Ti_on_Co_0 & 1.74786 & Co2ZnO4_V_on_Co_0 & 1.428 & 1.0342 & 0.71366 \\
Co2ZnO4_Ti_on_Co_-1 & 3.98017 & Co2ZnO4_V_on_Co_-1 & 1.428 & 3.10042 & 0.87975 \\
\hline Co2ZnO4_Cr_on_Zn_0 & 2.32195 & Co2ZnO4_V_on_Zn_0 & 3.69606 & 2.91725 & -0.5953 \\
Co2ZnO4_Cr_on_Zn_-1 & 4.07391 & Co2ZnO4_V_on_Zn_-1 & 3.69606 & 4.83029 & -0.75638 \\
\hline
\end{tabular}

Table 16: The difference between f-enthalpy values for a compound and its nearest neighbor, both with charge states 0 , and the corresponding compounds with charge state -1 (which are also nearest neighbors). Note the similarity in the differences, indicating that if three of the values are known, the fourth could be predicted. Compounds obtained from $\mathrm{Co}_{2} \mathrm{ZnO} 4$. 
B Results for the analysis of properties data for $\mathrm{Rh} 2 \mathrm{ZnO} 4$ 


\begin{tabular}{|c|c|c|c|c|}
\hline \multirow{2}{*}{ Compound ID } & \multicolumn{2}{|c|}{ nearest neighbor } & \multicolumn{2}{c|}{ farthest neighbor } \\
\cline { 2 - 6 } & compound & distance & compound & distance \\
\hline Rh2ZnO4_Zn_vacancy_0 & Rh2ZnO4_O_vacancy_0 & 3 & Rh2ZnO4_Ba_on_Rh_0 & 30.8605 \\
\hline Rh2ZnO4_Rh_vacancy_0 & Rh2ZnO4_O_vacancy_0 & 3 & Rh2ZnO4_Ba_on_Rh_0 & 32.1894 \\
\hline Rh2ZnO4_Zn_on_Rh_0 & Rh2ZnO4_Cr_on_Rh_0 & 3.88682 & Rh2ZnO4_Ba_on_Rh_0 & 28.1569 \\
\hline Rh2ZnO4_Rh_on_Zn_0 & Rh2ZnO4_Cr_on_Zn_0 & 2.65296 & Rh2ZnO4_Ba_on_Rh_0 & 30.8722 \\
\hline Rh2ZnO4_O_vacancy_0 & Rh2ZnO4_Cr_on_Rh_0 & 1.927 & Rh2ZnO4_Ba_on_Rh_0 & 30.7468 \\
\hline Rh2ZnO4_Be_on_Zn_0 & Rh2ZnO4_Mg_on_Zn_0 & 5.2123 & Rh2ZnO4_Ba_on_Rh_0 & 31.5509 \\
\hline Rh2ZnO4_Be_on_Rh_0 & Rh2ZnO4_Mg_on_Rh_0 & 10.7497 & Rh2ZnO4_Ba_on_Rh_0 & 32.4534 \\
\hline Rh2ZnO4_Mg_on_Zn_0 & Rh2ZnO4_Ca_on_Zn_0 & 4.21729 & Rh2ZnO4_Ba_on_Rh_0 & 31.09 \\
\hline Rh2ZnO4_Mg_on_Rh_0 & Rh2ZnO4_Zn_on_Rh_0 & 8.11697 & Rh2ZnO4_Ba_on_Rh_0 & 23.5869 \\
\hline Rh2ZnO4_Ca_on_Zn_0 & Rh2ZnO4_Sr_on_Zn_0 & 3.72967 & Rh2ZnO4_Ba_on_Rh_0 & 31.3335 \\
\hline Rh2ZnO4_Ca_on_Rh_0 & Rh2ZnO4_Sr_on_Rh_0 & 6.63866 & Rh2ZnO4_Ba_on_Zn_0 & 22.9136 \\
\hline Rh2ZnO4_Sr_on_Zn_0 & Rh2ZnO4_Ca_on_Zn_0 & 3.72967 & Rh2ZnO4_Ba_on_Rh_0 & 31.4753 \\
\hline Rh2ZnO4_Sr_on_Rh_0 & Rh2ZnO4_Ca_on_Rh_0 & 6.63866 & Rh2ZnO4_Ba_on_Zn_0 & 26.1638 \\
\hline Rh2ZnO4_Ba_on_Zn_0 & Rh2ZnO4_Sr_on_Zn_0 & 3.74677 & Rh2ZnO4_Ba_on_Rh_0 & 32.2407 \\
\hline Rh2ZnO4_Ba_on_Rh_0 & Rh2ZnO4_Sr_on_Rh_0 & 7.37422 & Rh2ZnO4_Be_on_Rh_0 & 32.4534 \\
\hline Rh2ZnO4_Ti_on_Zn_0 & Rh2ZnO4_V_on_Zn_0 & 1.64389 & Rh2ZnO4_Ba_on_Rh_0 & 31.1695 \\
\hline Rh2ZnO4_Ti_on_Rh_0 & Rh2ZnO4_V_on_Rh_0 & 2.12727 & Rh2ZnO4_Ba_on_Rh_0 & 27.3853 \\
\hline Rh2ZnO4_V_on_Zn_0 & Rh2ZnO4_Ti_on_Zn_0 & 1.64389 & Rh2ZnO4_Ba_on_Rh_0 & 30.9844 \\
\hline Rh2ZnO4_V_on_Rh_0 & Rh2ZnO4_Ti_on_Rh_0 & 2.12727 & Rh2ZnO4_Ba_on_Rh_0 & 27.8151 \\
\hline Rh2ZnO4_Cr_on_Zn_0 & Rh2ZnO4_Rh_on_Zn_0 & 2.65296 & Rh2ZnO4_Ba_on_Rh_0 & 31.1771 \\
\hline Rh2ZnO4_Cr_on_Rh_0 & Rh2ZnO4_O_vacancy_0 & 1.927 & Rh2ZnO4_Ba_on_Rh_0 & 30.2496 \\
\hline
\end{tabular}

Table 17: The distances to the nearest and farthest neighbors for the 21 compounds, with charge state o, obtained from Rh2ZnO4. 


\begin{tabular}{|c|c|c|c|c|}
\hline \multirow[t]{2}{*}{ Compound ID } & \multirow[t]{2}{*}{ f-enthalpy } & \multicolumn{3}{|c|}{ top two nearest neighbors } \\
\hline & & compound & distance & f-enthalpy \\
\hline \multirow[t]{2}{*}{ Rh2ZnO4_Zn_vacancy_0 } & 1.91477 & Rh2ZnO4_O_vacancy_0 & 3 & 2.83997 \\
\hline & & Rh2ZnO4_Cr_on_Rh_0 & 3.27312 & -2.28575 \\
\hline \multirow[t]{2}{*}{ Rh2ZnO4_Rh_vacancy_0 } & 2.08431 & Rh2ZnO4_O_vacancy_0 & 3 & 2.83997 \\
\hline & & Rh2ZnO4_Zn_vacancy_0 & 4 & 1.91477 \\
\hline \multirow[t]{2}{*}{ Rh2ZnO4_Zn_on_Rh_0 } & 0.531288 & Rh2ZnO4_Cr_on_Rh_0 & 3.88682 & -2.28575 \\
\hline & & Rh2ZnO4_O_vacancy_0 & 4.39799 & 2.83997 \\
\hline \multirow[t]{2}{*}{ Rh2ZnO4_Rh_on_Zn_0 } & 1.74137 & Rh2ZnO4_Cr_on_Zn_0 & 2.65296 & 0.231912 \\
\hline & & Rh2ZnO4_O_vacancy_0 & 3.11859 & 2.83997 \\
\hline \multirow[t]{2}{*}{ Rh2ZnO4_O_vacancy_0 } & 2.83997 & Rh2ZnO4_Cr_on_Rh_0 & 1.927 & -2.28575 \\
\hline & & Rh2ZnO4_Zn_vacancy_0 & 3 & 1.91477 \\
\hline \multirow[t]{2}{*}{ Rh2ZnO4_Be_on_Zn_0 } & 1.11011 & Rh2ZnO4_Mg_on_Zn_0 & 5.2123 & 0.018697 \\
\hline & & Rh2ZnO4_O_vacancy_0 & 7.21738 & 2.83997 \\
\hline \multirow[t]{2}{*}{ Rh2ZnO4_Be_on_Rh_0 } & 1.59864 & Rh2ZnO4_Mg_on_Rh_0 & 10.7497 & 0.123696 \\
\hline & & Rh2ZnO4_Cr_on_Rh_0 & 10.7591 & -2.28575 \\
\hline \multirow[t]{2}{*}{ Rh2ZnO4_Mg_on_Zn_0 } & 0.018697 & Rh2ZnO4_Ca_on_Zn_0 & 4.21729 & 0.306219 \\
\hline & & Rh2ZnO4_Rh_on_Zn_0 & 4.7415 & 1.74137 \\
\hline \multirow[t]{2}{*}{ Rh2ZnO4_Mg_on_Rh_0 } & 0.123696 & Rh2ZnO4_Zn_on_Rh_0 & 8.11697 & 0.531288 \\
\hline & & Rh2ZnO4_V_on_Rh_0 & 9.25622 & 0.778359 \\
\hline \multirow[t]{2}{*}{ Rh2ZnO4_Ca_on_Zn_0 } & 0.306219 & Rh2ZnO4_Sr_on_Zn_0 & 3.72967 & 0.875269 \\
\hline & & Rh2ZnO4_Mg_on_Zn_0 & 4.21729 & 0.018697 \\
\hline \multirow[t]{2}{*}{ Rh2ZnO4_Ca_on_Rh_0 } & 0.513393 & Rh2ZnO4_Sr_on_Rh_0 & 6.63866 & 1.39012 \\
\hline & & Rh2ZnO4_Mg_on_Rh_0 & 11.6526 & 0.123696 \\
\hline \multirow[t]{2}{*}{ Rh2ZnO4_Sr_on_Zn_0 } & 0.875269 & Rh2ZnO4_Ca_on_Zn_0 & 3.72967 & 0.306219 \\
\hline & & Rh2ZnO4_Ba_on_Zn_0 & 3.74677 & 1.89328 \\
\hline \multirow[t]{2}{*}{ Rh2ZnO4_Sr_on_Rh_0 } & 1.39012 & Rh2ZnO4_Ca_on_Rh_0 & 6.63866 & 0.513393 \\
\hline & & Rh2ZnO4_Ba_on_Rh_0 & 7.37422 & 2.872 \\
\hline \multirow[t]{2}{*}{ Rh2ZnO4_Ba_on_Zn_0 } & 1.89328 & Rh2ZnO4_Sr_on_Zn_0 & 3.74677 & 0.875269 \\
\hline & & Rh2ZnO4_Ca_on_Zn_0 & 7.32168 & 0.306219 \\
\hline \multirow[t]{2}{*}{ Rh2ZnO4_Ba_on_Rh_0 } & 2.872 & Rh2ZnO4_Sr_on_Rh_0 & 7.37422 & 1.39012 \\
\hline & & Rh2ZnO4_Ca_on_Rh_0 & 13.7106 & 0.513393 \\
\hline \multirow[t]{2}{*}{ Rh2ZnO4_Ti_on_Zn_0 } & 4.15957 & Rh2ZnO4_V_on_Zn_0 & 1.64389 & 3.51051 \\
\hline & & Rh2ZnO4_Rh_on_Zn_0 & 4.88414 & 1.74137 \\
\hline \multirow[t]{2}{*}{ Rh2ZnO4_Ti_on_Rh_0 } & 1.59054 & Rh2ZnO4_V_on_Rh_0 & 2.12727 & 0.778359 \\
\hline & & Rh2ZnO4_Cr_on_Rh_0 & 5.77581 & -2.28575 \\
\hline \multirow[t]{2}{*}{ Rh2ZnO4_V_on_Zn_0 } & 3.51051 & Rh2ZnO4_Ti_on_Zn_0 & 1.64389 & 4.15957 \\
\hline & & Rh2ZnO4_Rh_on_Zn_0 & 3.27032 & 1.74137 \\
\hline \multirow[t]{2}{*}{ Rh2ZnO4_V_on_Rh_0 } & 0.778359 & Rh2ZnO4_Ti_on_Rh_0 & 2.12727 & 1.59054 \\
\hline & & Rh2ZnO4_Cr_on_Rh_0 & 3.84245 & -2.28575 \\
\hline \multirow[t]{2}{*}{ Rh2ZnO4_Cr_on_Zn_0 } & 0.231912 & Rh2ZnO4_Rh_on_Zn_0 & 2.65296 & 1.74137 \\
\hline & & Rh2ZnO4_V_on_Zn_0 & 3.69606 & 3.51051 \\
\hline \multirow[t]{2}{*}{ Rh2ZnO4_Cr_on_Rh_0 } & -2.28575 & Rh2ZnO4_O_vacancy_0 & 1.927 & 2.83997 \\
\hline & & Rh2ZnO4_Zn_vacancy_0 & 3.27312 & 1.91477 \\
\hline
\end{tabular}

Table 18: The distances to the nearest two neighbors, and their f-enthalpy values, for the 21 compounds, with charge state 0, obtained from $\mathrm{Rh}_{2} \mathrm{ZnO} 4$. 


\begin{tabular}{|c|c|c|c|c|}
\hline \multirow{2}{*}{ Compound ID } & \multicolumn{2}{|c|}{ nearest neighbor } & \multicolumn{2}{c|}{ farthest neighbor } \\
\cline { 2 - 5 } & compound & distance & compound & distance \\
\hline Rh2ZnO4_Zn_vacancy_-1 & Rh2ZnO4_Cr_on_Rh_-1 & 3.27312 & Rh2ZnO4_Ba_on_Rh_-1 & 30.8605 \\
\hline Rh2ZnO4_Rh_vacancy_-1 & Rh2ZnO4_Zn_vacancy_-1 & 4 & Rh2ZnO4_Ba_on_Rh_-1 & 32.1894 \\
\hline Rh2ZnO4_Zn_on_Rh_-1 & Rh2ZnO4_Cr_on_Rh_-1 & 3.88682 & Rh2ZnO4_Ba_on_Rh_-1 & 28.1569 \\
\hline Rh2ZnO4_Be_on_Rh_-1 & Rh2ZnO4_Mg_on_Rh_-1 & 10.7497 & Rh2ZnO4_Ba_on_Rh_-1 & 32.4534 \\
\hline Rh2ZnO4_Mg_on_Rh_-1 & Rh2ZnO4_Zn_on_Rh_-1 & 8.11697 & Rh2ZnO4_Ba_on_Rh_-1 & 23.5869 \\
\hline Rh2ZnO4_Ca_on_Rh_-1 & Rh2ZnO4_Sr_on_Rh_-1 & 6.63866 & Rh2ZnO4_Rh_vacancy_-1 & 22.32 \\
\hline Rh2ZnO4_Sr_on_Rh_-1 & Rh2ZnO4_Ca_on_Rh_-1 & 6.63866 & Rh2ZnO4_Be_on_Rh_-1 & 25.9883 \\
\hline Rh2ZnO4_Ba_on_Rh_-1 & Rh2ZnO4_Sr_on_Rh_-1 & 7.37422 & Rh2ZnO4_Be_on_Rh_-1 & 32.4534 \\
\hline Rh2ZnO4_Ti_on_Zn_-1 & Rh2ZnO4_V_on_Zn_-1 & 1.64389 & Rh2ZnO4_Ba_on_Rh_-1 & 31.1695 \\
\hline Rh2ZnO4_Ti_on_Rh_-1 & Rh2ZnO4_V_on_Rh_-1 & 2.12727 & Rh2ZnO4_Ba_on_Rh_-1 & 27.3853 \\
\hline Rh2ZnO4_V_on_Zn_-1 & Rh2ZnO4_Ti_on_Zn_-1 & 1.64389 & Rh2ZnO4_Ba_on_Rh_-1 & 30.9844 \\
\hline Rh2ZnO4_V_on_Rh_-1 & Rh2ZnO4_Ti_on_Rh_-1 & 2.12727 & Rh2ZnO4_Ba_on_Rh_-1 & 27.8151 \\
\hline Rh2ZnO4_Cr_on_Zn_-1 & Rh2ZnO4_V_on_Zn_-1 & 3.69606 & Rh2ZnO4_Ba_on_Rh_-1 & 31.1771 \\
\hline Rh2ZnO4_Cr_on_Rh_-1 & Rh2ZnO4_Zn_vacancy_-1 & 3.27312 & Rh2ZnO4_Ba_on_Rh_-1 & 30.2496 \\
\hline
\end{tabular}

Table 19: The distances to the nearest and farthest neighbors for the 14 compounds, with charge state -1, obtained from $\mathrm{Rh} 2 \mathrm{ZnO}$. 


\begin{tabular}{|c|c|c|c|c|}
\hline \multirow[t]{2}{*}{ Compound ID } & \multirow[t]{2}{*}{ f-enthalpy } & \multicolumn{3}{|c|}{ top two nearest neighbors } \\
\hline & & compound & distance & f-enthalpy \\
\hline \multirow[t]{2}{*}{ Rh2ZnO4_Zn_vacancy_-1 } & 12.2039 & Rh2ZnO4_Cr_on_Rh_-1 & 3.27312 & 0.209424 \\
\hline & & Rh2ZnO4_Rh_vacancy_-1 & 4 & 2.38295 \\
\hline \multirow[t]{2}{*}{ Rh2ZnO4_Rh_vacancy_-1 } & 2.38295 & Rh2ZnO4_Zn_vacancy_-1 & 4 & 12.2039 \\
\hline & & Rh2ZnO4_Cr_on_Rh_-1 & 4.34778 & 0.209424 \\
\hline \multirow[t]{2}{*}{ Rh2ZnO4_Zn_on_Rh_-1 } & 0.694745 & Rh2ZnO4_Cr_on_Rh_-1 & 3.88682 & 0.209424 \\
\hline & & Rh2ZnO4_V_on_Rh_-1 & 4.87651 & 3.13748 \\
\hline \multirow[t]{2}{*}{ Rh2ZnO4_Be_on_Rh_-1 } & 1.7308 & Rh2ZnO4_Mg_on_Rh_-1 & 10.7497 & 0.494839 \\
\hline & & Rh2ZnO4_Cr_on_Rh_-1 & 10.7591 & 0.209424 \\
\hline \multirow[t]{2}{*}{ Rh2ZnO4_Mg_on_Rh_-1 } & 0.494839 & Rh2ZnO4_Zn_on_Rh_-1 & 8.11697 & 0.694745 \\
\hline & & Rh2ZnO4_V_on_Rh_-1 & 9.25622 & 3.13748 \\
\hline \multirow[t]{2}{*}{ Rh2ZnO4_Ca_on_Rh_-1 } & 1.14536 & Rh2ZnO4_Sr_on_Rh_-1 & 6.63866 & 2.0913 \\
\hline & & Rh2ZnO4_Mg_on_Rh_-1 & 11.6526 & 0.494839 \\
\hline \multirow[t]{2}{*}{ Rh2ZnO4_Sr_on_Rh_-1 } & 2.0913 & Rh2ZnO4_Ca_on_Rh_-1 & 6.63866 & 1.14536 \\
\hline & & Rh2ZnO4_Ba_on_Rh_-1 & 7.37422 & 3.5874 \\
\hline \multirow[t]{2}{*}{ Rh2ZnO4_Ba_on_Rh_-1 } & 3.5874 & Rh2ZnO4_Sr_on_Rh_-1 & 7.37422 & 2.0913 \\
\hline & & Rh2ZnO4_Ca_on_Rh_-1 & 13.7106 & 1.14536 \\
\hline \multirow[t]{2}{*}{ Rh2ZnO4_Ti_on_Zn_-1 } & 6.5012 & Rh2ZnO4_V_on_Zn_-1 & 1.64389 & 5.61178 \\
\hline & & Rh2ZnOH_Cr_on_Zn_-1 & 5.02217 & 2.58894 \\
\hline \multirow[t]{2}{*}{ Rh2ZnO4_Ti_on_Rh_-1 } & 4.40298 & Rh2ZnO4_V_on_Rh_-1 & 2.12727 & 3.13748 \\
\hline & & Rh2ZnO4_Cr_on_Rh_-1 & 5.77581 & 0.209424 \\
\hline \multirow[t]{2}{*}{ Rh2ZnO4_V_on_Zn_-1 } & 5.61178 & Rh2ZnO4_Ti_on_Zn_-1 & 1.64389 & 6.5012 \\
\hline & & Rh2ZnO4_Cr_on_Zn_-1 & 3.69606 & 2.58894 \\
\hline \multirow[t]{2}{*}{ Rh2ZnO4_V_on_Rh_-1 } & 3.13748 & Rh2ZnO4_Ti_on_Rh_-1 & 2.12727 & 4.40298 \\
\hline & & Rh2ZnO4_Cr_on_Rh_-1 & 3.84245 & 0.209424 \\
\hline \multirow[t]{2}{*}{ Rh2ZnO4_Cr_on_Zn_-1 } & 2.58894 & Rh2ZnO4_V_on_Zn_-1 & 3.69606 & 5.61178 \\
\hline & & Rh2ZnO4_Ti_on_Zn_-1 & 5.02217 & 6.5012 \\
\hline \multirow[t]{2}{*}{ Rh2ZnO4_Cr_on_Rh_-1 } & 0.209424 & Rh2ZnO4_Zn_vacancy_-1 & 3.27312 & 12.2039 \\
\hline & & Rh2ZnO4_V_on_Rh_-1 & 3.84245 & 3.13748 \\
\hline
\end{tabular}

Table 20: The distances to the nearest two neighbors, and their f-enthalpy values, for the 14 compounds, with charge state -1, obtained from $\mathrm{Rh}_{2} \mathrm{ZnO} 4$. 


\begin{tabular}{|c|c|c|c|c|c|}
\hline \multirow{2}{*}{ Compound ID } & \multirow{2}{*}{ f-enthalpy } & \multicolumn{4}{|c|}{ nearest neighbor } \\
\cline { 3 - 6 } & & compound & distance & f-enthalpy & difference \\
\hline Rh2ZnO4_Zn_on_Rh_0 & 0.531288 & Rh2ZnO4_Cr_on_Rh_0 & 3.88682 & -2.28575 & 2.817038 \\
Rh2ZnO4_Zn_on_Rh_-1 & 0.694745 & Rh2ZnO4_Cr_on_Rh_-1 & 3.88682 & 0.209424 & 0.485321 \\
\hline Rh2ZnO4_Be_on_Rh_0 & 1.59864 & Rh2ZnO4_Mg_on_Rh_0 & 10.7497 & 0.123696 & 1.474944 \\
Rh2ZnO4_Be_on_Rh_-1 & 1.7308 & Rh2ZnO4_Mg_on_Rh_-1 & 10.7497 & 0.494839 & 1.235961 \\
\hline Rh2ZnO4_Mg_on_Rh_0 & 0.123696 & Rh2ZnO4_Zn_on_Rh_0 & 8.11697 & 0.531288 & -0.407592 \\
Rh2ZnO4_Mg_on_Rh_-1 & 0.494839 & Rh2ZnO4_Zn_on_Rh_-1 & 8.11697 & 0.694745 & -0.199906 \\
\hline Rh2ZnO4_Ca_on_Rh_0 & 0.513393 & Rh2ZnO4_Sr_on_Rh_0 & 6.63866 & 1.39012 & -0.876727 \\
Rh2ZnO4_Ca_on_Rh_-1 & 1.14536 & Rh2ZnO4_Sr_on_Rh_-1 & 6.63866 & 2.0913 & -0.94594 \\
\hline Rh2ZnO4_Ti_on_Zn_0 & 4.15957 & Rh2ZnO4_V_on_Zn_0 & 1.64389 & 3.51051 & 0.64906 \\
Rh2ZnO4_Ti_on_Zn_-1 & 6.5012 & Rh2ZnO4_V_on_Zn_-1 & 1.64389 & 5.61178 & 0.88942 \\
\hline Rh2ZnO4_Ti_on_Rh_0 & 1.59054 & Rh2ZnO4_V_on_Rh_0 & 2.12727 & 0.778359 & 0.812181 \\
Rh2ZnO4_Ti_on_Rh_-1 & 4.40298 & Rh2ZnO4_V_on_Rh_-1 & 2.12727 & 3.13748 & 1.2655 \\
\hline
\end{tabular}

Table 21: The difference between f-enthalpy values for a compound and its nearest neighbor, both with charge states 0 , and the corresponding compounds with charge state -1 (which are also nearest neighbors). Note the similarity in the differences, indicating that if three of the values are known, the fourth could be predicted. Compounds obtained from Rh2ZnO4. 
C Results for the analysis of properties data for $\mathrm{Mn} 2 \mathrm{CrO} 4$ 


\begin{tabular}{|c|c|c|c|c|}
\hline \multirow[t]{2}{*}{ Compound ID } & \multicolumn{2}{|c|}{ nearest neighbor } & \multicolumn{2}{|c|}{ farthest neighbor } \\
\hline & compound & distance & compound & distance \\
\hline $\mathrm{Mn} 2 \mathrm{CrO} 4 \_\mathrm{Mn} \_$on_Cr_0 & Mn2CrO4_Cr_on_Mn_0 & 11.1046 & Mn2CrO4_Li__on_Mn_0 & 18.4639 \\
\hline Mn2CrO4_Ag_on_Mn_0 & Mn2CrO4_Cu_on_Mn_0 & 0.701159 & Mn2CrO4_Li_on_Mn_0 & 15.2968 \\
\hline Mn2CrO4_Cu_on_Mn_0 & Mn2CrO4_Cr_on_Mn_0 & 0.692069 & Mn2CrO4_Li_on_Mn_0 & 14.988 \\
\hline Mn2CrO4_Na_on_Cr_0 & Mn2CrO4_K_on_Cr_0 & 1.95465 & Mn2CrO4_Li_on_Mn_0 & 16.4218 \\
\hline Mn2CrO4_Li_on_Cr_0 & Mn2CrO4_Na_on_Cr_O & 8.19348 & Mn2CrO4_Li_on_Mn_0 & 20.4275 \\
\hline Mn2CrO4_Mn_vacancy_0 & $\mathrm{Mn} 2 \mathrm{CrO} 4 \_\mathrm{Cr} \_\mathrm{on} \_\mathrm{Mn} \_0$ & 2.46979 & Mn2CrO4_Li__on_Mn_0 & 16.3904 \\
\hline Mn2CrO4_Zn_on_Cr_0 & Mn2CrO4_Ag_on_Cr_0 & 1.93871 & Mn2CrO4_Li_on_Mn_0 & 14.9409 \\
\hline Mn2CrO4_Ag_on_Cr_0 & $\mathrm{Mn} 2 \mathrm{CrO} 4 \_\mathrm{Cu} \_$on_Cr_0 & 0.776023 & Mn2CrO4_Li__on_Mn_0 & 14.8787 \\
\hline Mn2CrO4_Cr_on_Mn_0 & Mn2CrO4_Cu_on_Mn_0 & 0.692069 & Mn2CrO4_Li_on_Mn_0 & 14.7162 \\
\hline Mn2CrO4_O_vacancy_0 & Mn2CrO4_Cr_on_Mn_0 & 1.4017 & Mn2CrO4_Li_on_Mn_0 & 14.8178 \\
\hline Mn2CrO4_Li_on_Mn_0 & Mn2CrO4_Na_On_Mn_0 & 8.49961 & Mn2CrO4_Li_on_Cr_0 & 20.4275 \\
\hline Mn2CrO4_Na_on_Mn_0 & Mn2CrO4_K_on_Mn_0 & 1.82446 & Mn2CrO4_Li_on_Cr_0 & 15.8181 \\
\hline Mn2CrO4_Zn_on_Mn_0 & Mn2CrO4_Cu_on_Mn_0 & 1.34267 & Mn2CrO4_Li_on_Mn_0 & 14.778 \\
\hline Mn2CrO4_Rb_on_Mn_0 & Mn2CrO4_Mg_on_Mn_0 & 3.78757 & Mn2CrO4_Li_on_Cr_0 & 15.189 \\
\hline Mn2CrO4_Mg_on_Cr_0 & Mn2CrO4_ZZn_on_Cr_0 & 3.43517 & Mn2CrO4_Li_on_Mn_0 & 15.26 \\
\hline Mn2CrO4_Mg_on_Mn_0 & Mn2CrO4_Na_On_Mn_0 & 3.57305 & Mn2CrO4_Li_on_Cr_0 & 14.5951 \\
\hline Mn2CrO4_Cr_vacancy_0 & $\mathrm{Mn} 2 \mathrm{CrO} 4 \_\mathrm{Cu} \_$on_Cr_0 & 2.97627 & Mn2CrO4_Li_on_Cr_0 & 15.8487 \\
\hline Mn2CrO4_K_on_Cr_0 & Mn2CrO4_Na_on_Cr_0 & 1.95465 & Mn2CrO4_Li_on_Mn_0 & 17.0221 \\
\hline Mn2CrO4_Rb_on_Cr_0 & Mn2CrO4_K_on_Cr_0 & 4.15801 & Mn2CrO4_Li_on_Mn_0 & 16.1827 \\
\hline Mn2CrO4_K_on_Mn_0 & Mn2CrO4_Na_On_Mn_0 & 1.82446 & Mn2CrO4_Li_on_Cr_0 & 16.3614 \\
\hline Mn2CrO4_Cu_on_Cr_0 & Mn2CrO4_Ag_on_Cr_0 & 0.776023 & Mn2CrO4_Li_on_Mn_0 & 14.8241 \\
\hline
\end{tabular}

Table 22: The distances to the nearest and farthest neighbors for the 21 compounds, with charge state 0 , obtained from $\mathrm{Mn} 2 \mathrm{CrO} 4$. 


\begin{tabular}{|c|c|c|c|c|}
\hline \multirow[t]{2}{*}{ Compound ID } & \multirow[t]{2}{*}{ f-enthalpy } & \multicolumn{3}{|c|}{ top two nearest neighbors } \\
\hline & & compound & distance & f-enthalpy \\
\hline \multirow[t]{2}{*}{ Mn2CrO4_Mn_on_Cr_0 } & \multirow[t]{2}{*}{1.11166} & Mn2CrO4_Cr_on_Mn_0 & 11.1046 & 0.921734 \\
\hline & & Mn2CrO4_O_vacancy_0 & 11.1062 & 5.01357 \\
\hline \multirow[t]{2}{*}{ Mn2CrO4_Ag_on_Mn_0 } & \multirow[t]{2}{*}{4.61637} & Mn2CrO4_Cu_on_Mn_0 & 0.701159 & 3.06201 \\
\hline & & Mn2CrO4_Cr_on_Mn_0 & 1.32404 & 0.921734 \\
\hline \multirow[t]{2}{*}{ Mn2CrO4_Cu_on_Mn_0 } & \multirow[t]{2}{*}{3.06201} & Mn2CrO4_Cr_on_Mn_0 & 0.692069 & 0.921734 \\
\hline & & Mn2CrO4_Ag_on_Mn_0 & 0.701159 & 4.61637 \\
\hline \multirow[t]{2}{*}{ Mn2CrO4_Na_on_Cr_0 } & \multirow[t]{2}{*}{10.9667} & Mn2CrO4_K_on_Cr_0 & 1.95465 & 5.07751 \\
\hline & & Mn2CrO4_Mg_on_Cr_0 & 3.51955 & -0.242771 \\
\hline \multirow[t]{2}{*}{ Mn2CrO4_Li_on_Cr_0 } & \multirow[t]{2}{*}{2.56028} & Mn2CrO4_Na_on_Cr_0 & 8.19348 & 10.9667 \\
\hline & & Mn2CrO4_K_on_Cr_0 & 8.5689 & 5.07751 \\
\hline \multirow[t]{2}{*}{ Mn2CrO4_Mn_vacancy_0 } & \multirow[t]{2}{*}{5.64584} & Mn2CrO4_Cr_on_Mn_0 & 2.46979 & 0.921734 \\
\hline & & Mn2CrO4_Cu_on_Mn_0 & 2.67209 & 3.06201 \\
\hline \multirow[t]{2}{*}{ Mn2CrO4_Zn_on_Cr_0 } & \multirow[t]{2}{*}{2.64323} & Mn2CrO4_Ag_on_Cr_0 & 1.93871 & 6.51794 \\
\hline & & Mn2CrO4_Cu_on_Cr_0 & 1.95502 & 4.64778 \\
\hline \multirow[t]{2}{*}{ Mn2CrO4_Ag_on_Cr_0 } & \multirow[t]{2}{*}{6.51794} & Mn2CrO4_Cu_on_Cr_0 & 0.776023 & 4.64778 \\
\hline & & Mn2CrO4_Zn_on_Cr_0 & 1.93871 & 2.64323 \\
\hline \multirow[t]{2}{*}{ Mn2CrO4_Cr_on_Mn_0 } & \multirow[t]{2}{*}{0.921734} & Mn2CrO4_Cu_on_Mn_0 & 0.692069 & 3.06201 \\
\hline & & Mn2CrO4_Ag_on_Mn_0 & 1.32404 & 4.61637 \\
\hline \multirow[t]{2}{*}{ Mn2CrO4_O_vacancy_0 } & \multirow[t]{2}{*}{5.01357} & Mn2CrO4_Cr_on_Mn_0 & 1.4017 & 0.921734 \\
\hline & & Mn2CrO4_Cu_on_Cr_0 & 1.4785 & 4.64778 \\
\hline \multirow[t]{2}{*}{ Mn2CrO4_Li_on_Mn_0 } & \multirow[t]{2}{*}{0.802801} & Mn2CrO4_Na_on_Mn_0 & 8.49961 & 9.07765 \\
\hline & & Mn2CrO4_K_on_Mn_0 & 8.76355 & 3.18072 \\
\hline Mn2CrO4_Na_on_Mn_0 & 9.07765 & Mn2CrO4_K_on_Mn_0 & 1.82446 & 3.18072 \\
\hline & & Mn2CrO4_Mg_on_Mn_0 & 3.57305 & -2.08582 \\
\hline Mn2CrO4_Zn_on_Mn_0 & 0.479527 & Mn2CrO4_Cu_on_Mn_0 & 1.34267 & 3.06201 \\
\hline & & Mn2CrO4_Ag_on_Mn_0 & 1.41205 & 4.61637 \\
\hline Mn2CrO4_Rb_on_Mn_0 & 4.32203 & Mn2CrO4_Mg_on_Mn_0 & 3.78757 & -2.08582 \\
\hline & & Mn2CrO4_K_on_Mn_0 & 4.17584 & 3.18072 \\
\hline Mn2CrO4_Mg_on_Cr_0 & -0.242771 & Mn2CrO4_Zn_on_Cr_0 & 3.43517 & 2.64323 \\
\hline & & Mn2CrO4_Na_on_Cr_0 & 3.51955 & 10.9667 \\
\hline Mn2CrO4_Mg_on_Mn_0 & -2.08582 & Mn2CrO4_Na_on_Mn_0 & 3.57305 & 9.07765 \\
\hline & & Mn2CrO4_Zn_on_Mn_0 & 3.578 & 0.479527 \\
\hline Mn2CrO4_Cr_vacancy_0 & 6.65839 & Mn2CrO4_Cu_on_Cr_0 & 2.97627 & 4.64778 \\
\hline & & Mn2CrO4_Cr_on_Mn_0 & 2.99412 & 0.921734 \\
\hline Mn2CrO4_K_on_Cr_0 & 5.07751 & Mn2CrO4_Na_on_Cr_0 & 1.95465 & 10.9667 \\
\hline & & Mn2CrO4_Rb_on_Cr_0 & 4.15801 & 6.39256 \\
\hline Mn2CrO4_Rb_on_Cr_0 & 6.39256 & Mn2CrO4_K_on_Cr_0 & 4.15801 & 5.07751 \\
\hline & & Mn2CrO4_Na_on_Cr_0 & 4.47093 & 10.9667 \\
\hline Mn2CrO4_K_on_Mn_0 & 3.18072 & Mn2CrO4_Na_on_Mn_0 & 1.82446 & 9.07765 \\
\hline & & Mn2CrO4_Rb_on_Mn_0 & 4.17584 & 4.32203 \\
\hline $\mathrm{Mn} 2 \mathrm{CrO} 4$ CU_on_Cr_0 & 4.64778 & Mn2CrO4_Ag_on_Cr_0 & 0.776023 & 6.51794 \\
\hline & & Mn2CrO4_Cr_on_Mn_0 & 1.46654 & 0.921734 \\
\hline
\end{tabular}

Table 23: The distances to the nearest two neighbors, and their f-enthalpy values, for the 21 compounds, with charge state 0, obtained from $\mathrm{Mn} 2 \mathrm{CrO} 4$. 


\begin{tabular}{|c|c|c|c|c|}
\hline \multirow{2}{*}{ Compound ID } & \multicolumn{2}{|c|}{ nearest neighbor } & \multicolumn{2}{c|}{ farthest neighbor } \\
\cline { 2 - 5 } & compound & distance & compound & distance \\
\hline Mn2CrO4_Mn_on_Cr_-1 & Mn2CrO4_Cu_on_Mn_-1 & 11.1185 & Mn2CrO4_Li_on_Mn_-1 & 18.4639 \\
\hline Mn2CrO4_Ag_on_Mn_-1 & Mn2CrO4_Cu_on_Mn_-1 & 0.701159 & Mn2CrO4_Li_on_Mn_-1 & 15.2968 \\
\hline Mn2CrO4_Cu_on_Mn_-1 & Mn2CrO4_Ag_on_Mn_-1 & 0.701159 & Mn2CrO4_Li_on_Mn_-1 & 14.988 \\
\hline Mn2CrO4_Na_on_Cr_-1 & Mn2CrO4_K_on_Cr_-1 & 1.95465 & Mn2CrO4_Li_on_Mn_-1 & 16.4218 \\
\hline Mn2CrO4_Li_on_Cr_-1 & Mn2CrO4_Na_on_Cr_-1 & 8.19348 & Mn2CrO4_Li_on_Mn_-1 & 20.4275 \\
\hline Mn2CrO4_Mn_vacancy_-1 & Mn2CrO4_Cu_on_Mn_-1 & 2.67209 & Mn2CrO4_Li_on_Mn_-1 & 16.3904 \\
\hline Mn2CrO4_Zn_on_Cr_-1 & Mn2CrO4_Ag_on_Cr_-1 & 1.93871 & Mn2CrO4_Li_on_Mn_-1 & 14.9409 \\
\hline Mn2CrO4_Ag_on_Cr_-1 & Mn2CrO4_Cu_on_Cr_-1 & 0.776023 & Mn2CrO4_Li_on_Mn_-1 & 14.8787 \\
\hline Mn2CrO4_Li_on_Mn_-1 & Mn2CrO4_Na_on_Mn_-1 & 8.49961 & Mn2CrO4_Li_on_Cr_-1 & 20.4275 \\
\hline Mn2CrO4_Na_on_Mn_-1 & Mn2CrO4_K_on_Mn_-1 & 1.82446 & Mn2CrO4_Li_on_Cr_-1 & 15.8181 \\
\hline Mn2CrO4_Rb_on_Mn_-1 & Mn2CrO4_K_on_Mn_-1 & 4.17584 & Mn2CrO4_Li_on_Cr_-1 & 15.189 \\
\hline Mn2CrO4_Mg_on_Cr_-1 & Mn2CrO4_Zn_on_Cr_-1 & 3.43517 & Mn2CrO4_Li_on_Mn_-1 & 15.26 \\
\hline Mn2CrO4_Cr_vacancy_-1 & Mn2CrO4_Cu_on_Cr_-1 & 2.97627 & Mn2CrO4_Li_on_Cr_-1 & 15.8487 \\
\hline Mn2CrO4_K_on_Cr_-1 & Mn2CrO4_Na_on_Cr_-1 & 1.95465 & Mn2CrO4_Li_on_Mn_-1 & 17.0221 \\
\hline Mn2CrO4_Rb_on_Cr_-1 & Mn2CrO4_K_on_Cr_-1 & 4.15801 & Mn2CrO4_Li_on_Mn_-1 & 16.1827 \\
\hline Mn2CrO4_K_on_Mn_-1 & Mn2CrO4_Na_on_Mn_-1 & 1.82446 & Mn2CrO4_Li_on_Cr_-1 & 16.3614 \\
\hline Mn2CrO4_Cu_on_Cr_-1 & Mn2CrO4_Ag_on_Cr_-1 & 0.776023 & Mn2CrO4_Li_on_Mn_-1 & 14.8241 \\
\hline
\end{tabular}

Table 24: The distances to the nearest and farthest neighbors for the 17 compounds, with charge state -1, obtained from $\mathrm{Mn} 2 \mathrm{CrO} 4$. 


\begin{tabular}{|c|c|c|c|c|}
\hline \multirow[t]{2}{*}{ Compound ID } & \multirow[t]{2}{*}{ f-enthalpy } & \multicolumn{3}{|c|}{ top two nearest neighbors } \\
\hline & & compound & distance & f-enthalpy \\
\hline \multirow[t]{2}{*}{ Mn2CrO4_Mn_on_Cr_-1 } & \multirow[t]{2}{*}{2.45537} & Mn2CrO4_Cu_on_Mn_-1 & 11.1185 & 4.02364 \\
\hline & & Mn2CrO4_Ag_on_Mn_-1 & 11.1646 & 4.98535 \\
\hline \multirow[t]{2}{*}{ Mn2CrO4_Ag_on_Mn_-1 } & \multirow[t]{2}{*}{4.98535} & Mn2CrO4_Cu_on_Mn_-1 & 0.701159 & 4.02364 \\
\hline & & Mn2CrO4_Cu_on_Cr_-1 & 1.86686 & 5.09537 \\
\hline \multirow[t]{2}{*}{ Mn2CrO4_Cu_on_Mn_-1 } & \multirow[t]{2}{*}{4.02364} & Mn2CrO4_Ag_on_Mn_-1 & 0.701159 & 4.98535 \\
\hline & & Mn2CrO4_Cu_on_Cr_-1 & 1.56778 & 5.09537 \\
\hline \multirow[t]{2}{*}{ Mn2CrO4_Na_on_Cr_-1 } & \multirow[t]{2}{*}{11.4986} & Mn2CrO4_K_on_Cr_-1 & 1.95465 & 5.70444 \\
\hline & & Mn2CrO4_Mg_on_Cr_-1 & 3.51955 & 0.019007 \\
\hline \multirow[t]{2}{*}{ Mn2CrO4_Li_on_Cr_-1 } & \multirow[t]{2}{*}{3.11996} & Mn2CrO4_Na_on_Cr_-1 & 8.19348 & 11.4986 \\
\hline & & Mn2CrO4_K_on_Cr_-1 & 8.5689 & 5.70444 \\
\hline \multirow[t]{2}{*}{ Mn2CrO4_Mn_vacancy_-1 } & \multirow[t]{2}{*}{6.00623} & Mn2CrO4_Cu_on_Mn_-1 & 2.67209 & 4.02364 \\
\hline & & Mn2CrO4_Ag_on_Mn_-1 & 2.83319 & 4.98535 \\
\hline \multirow[t]{2}{*}{ Mn2CrO4_Zn_on_Cr_-1 } & \multirow[t]{2}{*}{2.94207} & Mn2CrO4_Ag_on_Cr_-1 & 1.93871 & 7.24689 \\
\hline & & Mn2CrO4_Cu_on_Cr_-1 & 1.95502 & 5.09537 \\
\hline \multirow[t]{2}{*}{ Mn2CrO4_Ag_on_Cr_-1 } & \multirow[t]{2}{*}{7.24689} & Mn2CrO4_Cu_on_Cr_-1 & 0.776023 & 5.09537 \\
\hline & & Mn2CrO4_Zn_on_Cr_-1 & 1.93871 & 2.94207 \\
\hline \multirow[t]{2}{*}{ Mn2CrO4_Li_on_Mn_-1 } & \multirow[t]{2}{*}{0.995454} & Mn2CrO4_Na_on_Mn_-1 & 8.49961 & 9.29108 \\
\hline & & Mn2CrO4_K_on_Mn_-1 & 8.76355 & 3.45242 \\
\hline \multirow[t]{2}{*}{ Mn2CrO4_Na_on_Mn_-1 } & \multirow[t]{2}{*}{9.29108} & Mn2CrO4_K_on_Mn_-1 & 1.82446 & 3.45242 \\
\hline & & Mn2CrO4_Rb_on_Mn_-1 & 4.18869 & 4.67202 \\
\hline \multirow[t]{2}{*}{ Mn2CrO4_Rb_on_Mn_-1 } & \multirow[t]{2}{*}{4.67202} & Mn2CrO4_K_on_Mn_-1 & 4.17584 & 3.45242 \\
\hline & & Mn2CrO4_Na_on_Mn_-1 & 4.18869 & 9.29108 \\
\hline \multirow[t]{2}{*}{ Mn2CrO4_Mg_on_Cr_-1 } & \multirow[t]{2}{*}{0.019007} & Mn2CrO4_Zn_on_Cr_-1 & 3.43517 & 2.94207 \\
\hline & & Mn2CrO4_Na_on_Cr_-1 & 3.51955 & 11.4986 \\
\hline \multirow[t]{2}{*}{ Mn2CrO4_Cr_vacancy_-1 } & \multirow[t]{2}{*}{7.27288} & Mn2CrO4_Cu_on_Cr_-1 & 2.97627 & 5.09537 \\
\hline & & Mn2CrO4_Cu_on_Mn_-1 & 3.04499 & 4.02364 \\
\hline Mn2CrO4_K_on_Cr_-1 & 5.70444 & Mn2CrO4_Na_on_Cr_-1 & 1.95465 & 11.4986 \\
\hline & & Mn2CrO4_Rb_on_Cr_-1 & 4.15801 & 7.05915 \\
\hline Mn2CrO4_Rb_on_Cr_-1 & 7.05915 & Mn2CrO4_K_on_Cr_-1 & 4.15801 & 5.70444 \\
\hline & & Mn2CrO4_Na_on_Cr_-1 & 4.47093 & 11.4986 \\
\hline Mn2CrO4_K_on_Mn_-1 & 3.45242 & Mn2CrO4_Na_on_Mn_-1 & 1.82446 & 9.29108 \\
\hline & & Mn2CrO4_Rb_on_Mn_-1 & 4.17584 & 4.67202 \\
\hline Mn2CrO4_Cu_on_Cr_-1 & 5.09537 & Mn2CrO4_Ag_on_Cr_-1 & 0.776023 & 7.24689 \\
\hline & & Mn2CrO4_Cu_on_Mn_-1 & 1.56778 & 4.02364 \\
\hline
\end{tabular}

Table 25: The distances to the nearest two neighbors, and their f-enthalpy values, for the 17 compounds, with charge state -1, obtained from $\mathrm{Mn} 2 \mathrm{CrO} 4$. 


\begin{tabular}{|c|c|c|c|c|c|}
\hline \multirow[t]{2}{*}{ Compound ID } & \multirow[t]{2}{*}{ f-enthalpy } & \multicolumn{4}{|c|}{ nearest neighbor } \\
\hline & & compound & distance & f-enthalpy & difference \\
\hline Mn2CrO4_Ag_on_Mn_0 & 4.61637 & Mn2CrO4_Cu_on_Mn_0 & 0.701159 & 3.06201 & 1.55436 \\
\hline Mn2CrO4_Ag_on_Mn_-1 & 4.98535 & Mn2CrO4_Cu_on_Mn_-1 & 0.701159 & 4.02364 & 0.96171 \\
\hline Mn2CrO4_Na_on_Cr_0 & 10.9667 & Mn2CrO4_K_on_Cr_0 & 1.95465 & 5.07751 & 5.88919 \\
\hline Mn2CrO4_Na_on_Cr_-1 & 11.4986 & Mn2CrO4_K_on_Cr_-1 & 1.95465 & 5.70444 & 5.79416 \\
\hline Mn2CrO4_Li_on_Cr_0 & 2.56028 & $\mathrm{Mn} 2 \mathrm{CrO} 4 \_\mathrm{Na} \_$on_Cr_0 & 8.19348 & 10.9667 & -8.40642 \\
\hline Mn2CrO4_Li_on_Cr_-1 & 3.11996 & Mn2CrO4_Na_on_Cr_-1 & 8.19348 & 11.4986 & -8.37864 \\
\hline Mn2CrO4_Zn_on_Cr_0 & 2.64323 & Mn2CrO4_Ag_on_Cr_0 & 1.93871 & 6.51794 & -3.87471 \\
\hline Mn2CrO4_Zn_on_Cr_-1 & 2.94207 & Mn2CrO4_Ag_on_Cr_-1 & 1.93871 & 7.24689 & -4.30482 \\
\hline Mn2CrO4_Ag_on_Cr_0 & 6.51794 & $\mathrm{Mn} 2 \mathrm{CrO} 4 \_\mathrm{Cu} \_$on_Cr_0 & 0.776023 & 4.64778 & 1.87016 \\
\hline Mn2CrO4_Ag_on_Cr_-1 & 7.24689 & Mn2CrO4_Cu_on_Cr_-1 & 0.776023 & 5.09537 & 2.15153 \\
\hline Mn2CrO4_Li_on_Mn_0 & 0.802801 & Mn2CrO4_Na_on_Mn_0 & 8.49961 & 9.07765 & -8.274849 \\
\hline Mn2CrO4_Li_on_Mn__-1 & 0.995454 & Mn2CrO4_Na_on_Mn_-1 & 8.49961 & 9.29108 & -8.295626 \\
\hline Mn2CrO4_Na_on_Mn_0 & 9.07765 & Mn2CrO4_K_on_Mn_0 & 1.82446 & 3.18072 & 5.89693 \\
\hline Mn2CrO4_Na_on_Mn_-1 & 9.29108 & Mn2CrO4_K_on_Mn_-1 & 1.82446 & 3.45242 & 5.83866 \\
\hline Mn2CrO4_Mg_on_Cr_0 & -0.242771 & Mn2CrO4_Zn_on_Cr_0 & 3.43517 & 2.64323 & -2.886001 \\
\hline Mn2CrO4_Mg_on_Cr_-1 & 0.019007 & Mn2CrO4_Zn_on_Cr_-1 & 3.43517 & 2.94207 & -2.923063 \\
\hline Mn2CrO4_Cr_vacancy_0 & 6.65839 & $\mathrm{Mn} 2 \mathrm{CrO} 4 \_\mathrm{Cu}$ _on_Cr_0 & 2.97627 & 4.64778 & 2.01061 \\
\hline Mn2CrO4_Cr_vacancy_-1 & 7.27288 & Mn2CrO4_Cu_on_Cr_-1 & 2.97627 & 5.09537 & 2.17751 \\
\hline Mn2CrO4_Rb_on_Cr_0 & 6.39256 & Mn2CrO4_K_on_Cr_0 & 4.15801 & 5.07751 & 1.31505 \\
\hline Mn2CrO4_Rb_on_Cr_-1 & 7.05915 & Mn2CrO4_K_on_Cr_-1 & 4.15801 & 5.70444 & 1.35471 \\
\hline
\end{tabular}

Table 26: The difference between f-enthalpy values for a compound and its nearest neighbor, both with charge states 0 , and the corresponding compounds with charge state -1 (which are also nearest neighbors). Note the similarity in the differences, indicating that if three of the values are known, the fourth could be predicted. Compounds obtained from $\mathrm{Mn} 2 \mathrm{CrO} 4$. 